\title{
Symplectic Kloosterman sums and Poincaré series
}

\section{Siu Hang $\operatorname{Man}^{1}$}

Received: 5 June 2020 / Accepted: 25 July 2021 / Published online: 1 October 2021

(c) The Author(s) 2021

\section{Abstract}

We prove power-saving bounds for general Kloosterman sums on $\mathrm{Sp}(4)$ associated to all Weyl elements via a stratification argument coupled with $p$-adic stationary phase methods. We relate these Kloosterman sums to the Fourier coefficients of $\mathrm{Sp}(4)$ Poincare series.

Keywords Kloosterman sums · Poincaré series

Mathematics Subject Classification 11L05 · 11F30

\section{Introduction}

The classical Kloosterman sum is given by

$$
S(m, n ; q)=\sum_{\substack{x, y \in \mathbb{Z} / q \mathbb{Z} \\ x y \equiv 1}} \mathrm{e}\left(\frac{m x+n y}{q}\right),
$$

where e $(x)=e^{2 \pi i x}$. Kloosterman sums naturally appear in the Fourier expansion of GL(2) Poincaré series

$$
P_{m}(z ; v)=\sum_{\gamma \in \Gamma_{\infty} \backslash \operatorname{SL}(2, \mathbb{Z})} \operatorname{Im}(\gamma z)^{v} \mathrm{e}(m(\gamma z)),
$$

which play an important role in number theory. In [2], Bump, Friedberg and Goldfeld introduced GL( $r$ ) Poincaré series for $r \geq 2$, and gave a generalisation of Kloosterman

The author was supported in part by the DAAD Graduate School Scholarship Programme.

$凶 \quad$ Siu Hang Man

shman@math.uni-bonn.de

1 Mathematisches Institut, Rheinische Friedrich-Wilhelms-Universität Bonn, Endenicher Allee 60, 53115 Bonn, Germany 
sums to GL(3). The notion of Kloosterman sums was then generalised to GL( $r$ ) for $r \geq 2$ by Friedberg [9], and then to arbitrary simply connected Chevalley groups by Dąbrowski [5].

By methods of algebraic geometry, Weil [16] obtained a bound for GL(2) Kloosterman sums

$$
|S(m, n ; q)| \ll \tau(q)(m, n, q)^{1 / 2} q^{1 / 2},
$$

where $\tau$ denotes the divisor function. However, it remains a major open problem to give non-trivial bounds for Kloosterman sums in general, and currently only a small set of examples can be treated. Bounds for GL(3) Kloosterman sums were first obtained by Larsen [2, Appendix] and Stevens [14], and were improved by Dạbrowski and Fisher [7]. Bounds for GL(4) Kloosterman sums were given by Huang [10, Appendix]. Friedberg [9] generalised the results to GL( $r)$ Kloosterman sums attached to certain Weyl elements. On reductive groups, Dạbrowski and Reeder [8] gave the size of Kloosterman sets, establishing a trivial bound for Kloosterman sums on reductive groups.

Other than Poincaré series, another application of Kloosterman sums is found in the relative trace formula, which integrates an automorphic kernel over two subgroups with their respective characters. In particular, a prime application for bounds of Kloosterman sums is the analysis of the arithmetic side of the Petersson/Kuznetsov spectral summation formula. A more detailed description of this can be found in [4].

Now we introduce the main results. Let

$$
G=\operatorname{Sp}(2 r)=\left\{M \in \operatorname{GL}(2 r) \mid M^{T} J M=J\right\}, \quad J=\left(\begin{array}{c}
I_{n} \\
-I_{n}
\end{array}\right)
$$

be the standard symplectic group. When $k$ is a field, $G(k)$ is the group of linear transformations of $k^{2 r}$ preserving a symplectic bilinear form on $k^{2 r}$. The standard torus and the standard unipotent subgroup of $G$ are given by

$$
\begin{aligned}
& T=\left\{\left(\begin{array}{cc}
T_{0} & \\
& T_{0}^{-1}
\end{array}\right) \in G \mid T_{0} \text { diagonal }\right\} \\
& U=\left\{\left(\begin{array}{cc}
U_{0} & S \\
& \left(U_{0}^{-1}\right)^{T}
\end{array}\right) \in G \mid U_{0} \text { upper triangular, unipotent }\right\}
\end{aligned}
$$

respectively. We denote by $N=N_{G}(T)$ the normaliser of $T$ in $G$. The Weyl group is given by $W:=N_{G}(T) / T$. Let $w: N \rightarrow W$ be the canonical projection map with respect to this decomposition. For $n \in N$, we also define $U_{n}:=U \cap n^{-1} U^{T} n$, and $\bar{U}_{n}:=U \cap n^{-1} U n$. Note that $U_{n}, \bar{U}_{n}$ depend only on the image $w(n)$ of the canonical projection.

Here we follow the notations in Stevens [14]. Let $p$ be a rational prime. We have a Bruhat decomposition

$$
G\left(\mathbb{Q}_{p}\right)=U\left(\mathbb{Q}_{p}\right) N\left(\mathbb{Q}_{p}\right) U_{n}\left(\mathbb{Q}_{p}\right)
$$


For $n \in N\left(\mathbb{Q}_{p}\right)$, we define

$$
\begin{aligned}
& C(n)=U\left(\mathbb{Q}_{p}\right) n U\left(\mathbb{Q}_{p}\right) \cap G\left(\mathbb{Z}_{p}\right), \\
& X(n)=U\left(\mathbb{Z}_{p}\right) \backslash C(n) / U_{n}\left(\mathbb{Z}_{p}\right),
\end{aligned}
$$

and projection maps

$$
\begin{gathered}
u: X(n) \rightarrow U\left(\mathbb{Z}_{p}\right) \backslash U\left(\mathbb{Q}_{p}\right), \\
u^{\prime}: X(n) \rightarrow U\left(\mathbb{Q}_{p}\right) / U_{n}\left(\mathbb{Z}_{p}\right)
\end{gathered}
$$

by the relation $x=u(x) n u^{\prime}(x)$ for $x \in X(n)$.

Remark In [14], the notions above are defined for GL( $r)$, but it is straightforward to check that the construction also works for $\operatorname{Sp}(2 r)$, with essentially the same proofs. In particular, $X(n)$ is finite, and the projection maps $u, u^{\prime}$ are well-defined.

Let $n \in N\left(\mathbb{Q}_{p}\right), \psi_{p}$ a character of $U\left(\mathbb{Q}_{p}\right)$ which is trivial on $U\left(\mathbb{Z}_{p}\right)$, and $\psi_{p}^{\prime}$ a character of $U_{n}\left(\mathbb{Q}_{p}\right)$ trivial on $U_{n}\left(\mathbb{Z}_{p}\right)$, such that $\psi_{p}^{\prime}$ is the restriction of some character of $U\left(\mathbb{Q}_{p}\right)$ trivial on $U\left(\mathbb{Z}_{p}\right)$. Then the local Kloosterman sum is given by

$$
\mathrm{Kl}_{p}\left(n, \psi_{p}, \psi_{p}^{\prime}\right)=\sum_{x \in X(n)} \psi_{p}(u(x)) \psi_{p}^{\prime}\left(u^{\prime}(x)\right) .
$$

If $\psi_{p}^{\prime}$ is given as a character of $U\left(\mathbb{Q}_{p}\right)$ which is trivial on $U\left(\mathbb{Z}_{p}\right)$, we write $\mathrm{Kl}_{p}\left(n, \psi_{p}, \psi_{p}^{\prime}\right)$ to mean $\mathrm{Kl}_{p}\left(n, \psi_{p},\left.\psi_{p}^{\prime}\right|_{U_{n}\left(\mathbb{Q}_{p}\right)}\right)$.

To define a global Kloosterman sum, let $n \in N(\mathbb{Q}), \psi=\prod_{p} \psi_{p}$ a character of $U(\mathbb{A})$ which is trivial on $\prod_{p} U\left(\mathbb{Z}_{p}\right)$, and $\psi^{\prime}$ a character of $U_{n}(\mathbb{A})$ trivial on $\prod_{p} U_{n}\left(\mathbb{Z}_{p}\right)$, such that $\psi^{\prime}$ is the restriction of some character of $U(\mathbb{A})$ trivial on $\prod_{p} U\left(\mathbb{Z}_{p}\right)$. Then the global Kloosterman sum is given by

$$
\mathrm{Kl}_{p}\left(n, \psi, \psi^{\prime}\right)=\prod_{p} \mathrm{Kl}_{p}\left(n, \psi_{p}, \psi_{p}^{\prime}\right) .
$$

Remark This definition of Kloosterman sums is different from the symplectic Kloosterman sums introduced by Kitaoka [11], which are more relevant for classical Sp(4) Fourier expansions with respect to the upper right 2-by-2 block, which however is not a full parabolic subgroup. Tóth [15] proved some properties and estimates of such Kloosterman sums. The Kloosterman sums introduced here fit into the general framework of Kloosterman sums defined on reductive groups, see e.g. Dạbrowski [5].

For $G=\operatorname{Sp}\left(4, \mathbb{Q}_{p}\right)$, a set of simple roots of $G$ with respect to the maximal torus $T$ is given by $\Delta=\{\alpha, \beta\}$, where

$$
\alpha\left(\operatorname{diag}\left(y_{1}, y_{2}, y_{1}^{-1}, y_{2}^{-1}\right)\right)=y_{1} y_{2}^{-1}, \quad \beta\left(\operatorname{diag}\left(y_{1}, y_{2}, y_{1}^{-1}, y_{2}^{-1}\right)\right)=y_{2}^{2} .
$$


Then $\Psi^{+}=\{\alpha, \beta, \alpha+\beta, 2 \alpha+\beta\}$ is a set of positive roots. We denote by $s_{\alpha}$ and $s_{\beta}$ the simple reflections in the hyperplane orthogonal to $\alpha$ and $\beta$ respectively. Then the Weyl group of $G$ with respect to $T$ is given by

$$
W=\left\{1, s_{\alpha}, s_{\beta}, s_{\alpha} s_{\beta}, s_{\beta} s_{\alpha}, s_{\alpha} s_{\beta} s_{\alpha}, s_{\beta} s_{\alpha} s_{\beta}, s_{\alpha} s_{\beta} s_{\alpha} s_{\beta}\right\}
$$

We fix once and for all representatives for $s_{\alpha}$ and $s_{\beta}$ :

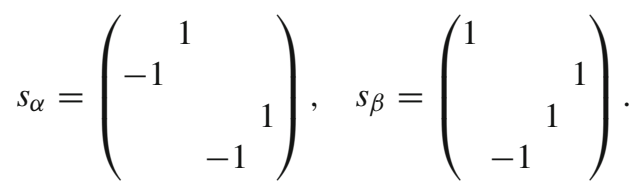

We also denote the long Weyl element $s_{\alpha} s_{\beta} s_{\alpha} s_{\beta}$ by $w_{0}$. Characters of $U\left(\mathbb{Q}_{p}\right)$ trivial on $U\left(\mathbb{Z}_{p}\right)$ are given by $\psi_{m_{1}, m_{2}}$ for $m_{1}, m_{2} \in \mathbb{Z}$, where

$$
\psi_{m_{1}, m_{2}}\left(\begin{array}{cccc}
1 & x_{1} & * & * \\
& 1 & * & x_{2} \\
& & 1 & \\
& & -x_{1} & 1
\end{array}\right)=\mathrm{e}\left(m_{1} x_{1}+m_{2} x_{2}\right)
$$

where e $: \mathbb{Q}_{p} / \mathbb{Z}_{p} \rightarrow \mathbb{C}^{\times}$is the standard additive character satisfying $\mathrm{e}\left(p^{-r}\right)$ $=e^{2 \pi i / p^{r}}$. For $w \in W, r, s \in \mathbb{Z}$, we set

$$
n_{w, r, s}:=\operatorname{diag}\left(p^{-r}, p^{r-s}, p^{r}, p^{s-r}\right) w \in N\left(\mathbb{Q}_{p}\right)
$$

The exact conditions $r, s$ have to satisfy for $X\left(n_{w, r, s}\right)$ to be nonempty are given in Sect. 3, but in general we require $r, s \geq 0$. By counting the number of terms in the Kloosterman sum [8, Theorem 0.3 , we obtain a trivial bound

$$
\left|\mathrm{Kl}_{p}\left(n_{w, r, s}, \psi, \psi^{\prime}\right)\right| \leq p^{r+s}
$$

Now we state the main results of the paper. The first result concerns non-trivial bounds for local $\mathrm{Sp}$ (4) Kloosterman sums. 
Theorem 1.1 Let $\psi=\psi_{m_{1}, m_{2}}, \psi^{\prime}=\psi_{n_{1}, n_{2}}$ be characters of $U\left(\mathbb{Q}_{p}\right) / U\left(\mathbb{Z}_{p}\right)$, and $r, s$ be non-negative integers. Then we have

$$
\begin{aligned}
& \mathrm{Kl}_{p}\left(n_{\mathrm{id}, r, s}, \psi, \psi^{\prime}\right)=1 \\
& \left|\mathrm{Kl}_{p}\left(n_{s_{\alpha}, r, s}, \psi, \psi^{\prime}\right)\right| \ll p^{r / 2}\left(m_{1}, n_{1}, p^{r}\right)^{1 / 2} \\
& \left|\mathrm{Kl}_{p}\left(n_{s_{\beta}, r, s}, \psi, \psi^{\prime}\right)\right| \ll p^{s / 2}\left(m_{2}, n_{2}, p^{s}\right)^{1 / 2} \\
& \left|\mathrm{Kl}_{p}\left(n_{s_{\alpha} s_{\beta}, r, s}, \psi, \psi^{\prime}\right)\right| \ll \min \left\{p^{2 s}\left(m_{1}, p^{r-s}\right), p^{r}\left(m_{2}, p^{s}\right)^{1 / 2}\left(n_{2}, p^{s}\right)^{1 / 2}\right\} \\
& \text { if } r=s=0 \text {, } \\
& \text { if } s=0 \text {, } \\
& \text { if } r=0 \text {, } \\
& \left|\mathrm{Kl}_{p}\left(n_{s_{\beta} s_{\alpha}, r, s}, \psi, \psi^{\prime}\right)\right| \ll \min \left\{p^{3 r}\left(m_{2}, p^{s-2 r}\right), p^{s}\left(m_{1}, n_{1}, p^{r}\right)\right\} \\
& \text { if } s \leq r \text {, } \\
& \left|\mathrm{Kl}_{p}\left(n_{s_{\alpha} s_{\beta} s_{\alpha}, r, s}, \psi, \psi^{\prime}\right)\right| \ll\left\{\begin{array}{l}
p^{\frac{r}{3}+\frac{2 s}{3}+\frac{2}{3} \min \left\{\operatorname{ord}_{p}\left(m_{1}\right)+s, \operatorname{ord}_{p}\left(n_{1}\right)+r\right\}+\frac{1}{3} \operatorname{ord}_{p}\left(m_{2}\right)} \\
p^{r+\min \left\{\operatorname{ord}_{p}\left(m_{2}\right), r+\operatorname{ord}_{p}\left(n_{1}\right)\right\}}+p^{r+\min \left\{\frac{s}{2}+\operatorname{ord}_{p}\left(m_{1}\right), r-\frac{s}{2}+\operatorname{ord}_{p}\left(n_{1}\right)\right\}} \\
p^{r+\min \left\{\operatorname{ord}_{p}\left(m_{2}\right), r+\operatorname{ord}_{p}\left(n_{1}\right)\right\}}
\end{array}\right. \\
& \text { if } 2 r \leq s \text {, } \\
& \left|\mathrm{Kl}_{p}\left(n_{s_{\beta} s_{\alpha} s_{\beta}, r, s}, \psi, \psi^{\prime}\right)\right| \ll \begin{cases}p^{\frac{s}{2}+\frac{r}{2}+\frac{1}{2} \operatorname{ord}_{p}\left(m_{1}\right)+\frac{1}{2} \min \left\{2 r+\operatorname{ord}_{p}\left(m_{2}\right), s+\operatorname{ord}_{p}\left(n_{2}\right)\right\},} & \text { if } r \leq s / 2, \\
p^{s-\frac{r}{2}+\frac{1}{2} \operatorname{ord}_{p}\left(m_{1}\right)+\frac{1}{2} \min \left\{2 r+\operatorname{ord}_{p}\left(m_{2}\right), s+\operatorname{ord}_{p}\left(n_{2}\right)\right\}} & \text { if } s / 2<r<s, \\
p^{s+\min \left\{\operatorname{ord}_{p}\left(m_{1}\right), \operatorname{ord}_{p}\left(n_{2}\right)\right\}} & \text { if } r=s,\end{cases} \\
& \left|\mathrm{Kl}_{p}\left(n_{w_{0}, r, s}, \psi, \psi^{\prime}\right)\right| \ll \min \left\{p^{\frac{1}{2} \operatorname{ord}_{p}\left(m_{1} m_{2}\right)}, p^{\frac{1}{2} \operatorname{ord}_{p}\left(n_{1} n_{2}\right)}\right\}(s+1) p^{\frac{r}{2}+\frac{3 s}{4}+\frac{1}{2} \min \{r, s\}} .
\end{aligned}
$$

Moreover, the Kloosterman sum $\mathrm{Kl}_{p}\left(n_{w, r, s}, \psi, \psi^{\prime}\right)$ vanishes if the conditions on the right are not satisfied.

Now we state the bounds for global $\mathrm{Sp}(4)$ Kloosterman sums. Recall that characters of $U(\mathbb{Q}) / U(\mathbb{Z})$ are given by $\psi_{m_{1}, m_{2}}$ for $m_{1}, m_{2} \in \mathbb{Z}$, where

$$
\psi_{m_{1}, m_{2}}\left(\begin{array}{cccc}
1 & x_{1} & * & * \\
& 1 & * & x_{2} \\
& & 1 & \\
& & -x_{1} & 1
\end{array}\right)=\mathrm{e}\left(m_{1} x_{1}+m_{2} x_{2}\right)
$$

For $w \in W, c_{1}, c_{2} \in \mathbb{N}$, let

$$
n_{w}\left(c_{1}, c_{2}\right):=\operatorname{diag}\left(c_{1}^{-1}, c_{1} c_{2}^{-1}, c_{1}, c_{1}^{-1} c_{2}\right) w \in N(\mathbb{Q}) .
$$


Theorem 1.2 Let $\psi=\psi_{m_{1}, m_{2}}, \psi^{\prime}=\psi_{n_{1}, n_{2}}$ be characters of $U(\mathbb{Q}) / U(\mathbb{Z})$. For every $\varepsilon>0$ and $c_{1}, c_{2} \in \mathbb{N}$, the following bounds hold:

$$
\begin{aligned}
\mathrm{Kl}\left(n_{\mathrm{id}}\left(c_{1}, c_{2}\right), \psi, \psi^{\prime}\right) & =1 & \text { if } c_{1}=c_{2} \\
\left|\mathrm{Kl}\left(n_{s_{\alpha}}\left(c_{1}, c_{2}\right), \psi, \psi^{\prime}\right)\right| & \ll_{\varepsilon}\left(m_{1}, n_{1}, c_{1}\right)^{1 / 2} c_{1}^{1 / 2+\varepsilon} & \text { if } c_{2}=1, \\
\left|\mathrm{Kl}\left(n_{s_{\beta}}\left(c_{1}, c_{2}\right), \psi, \psi^{\prime}\right)\right| & \ll_{\varepsilon}\left(m_{2}, n_{2}, c_{2}\right)^{1 / 2} c_{2}^{1 / 2+\varepsilon} & \text { if } c_{1}=1, \\
\left|\mathrm{Kl}\left(n_{s_{\alpha} s_{\beta}}\left(c_{1}, c_{2}\right), \psi, \psi^{\prime}\right)\right| & \ll_{\varepsilon}\left(c_{2}^{2}\left(m_{1}, c_{1} / c_{2}\right), c_{1}\left(m_{2}, c_{2}\right)^{1 / 2}\left(n_{2}, c_{2}\right)^{1 / 2}\right)\left(c_{1} c_{2}\right)^{\varepsilon} & \text { if } c_{2} \mid c_{1}, \\
\left|\mathrm{Kl}\left(n_{s_{\beta} s_{\alpha}}\left(c_{1}, c_{2}\right), \psi, \psi^{\prime}\right)\right| & \ll_{\varepsilon}\left(c_{1}^{3}\left(m_{2}, c_{2} / c_{1}^{2}\right), c_{2}\left(m_{1}, n_{1}, c_{1}\right)\right)\left(c_{1} c_{2}\right)^{\varepsilon} & \text { if } c_{1}^{2} \mid c_{2}, \\
\left|\mathrm{Kl}\left(n_{s_{\alpha} s_{\beta} s_{\alpha}}\left(c_{1}, c_{2}\right), \psi, \psi^{\prime}\right)\right| & \ll_{\varepsilon}\left(m_{1}, n_{1}, c_{1}\right)\left(m_{2}, c_{2}\right)\left(c_{1}, c_{2}\right)\left(c_{1} c_{2}\right)^{1 / 3+\varepsilon} & \text { if } c_{2} \mid c_{1}^{2}, \\
\left|\mathrm{Kl}\left(n_{s_{\beta} s_{\alpha} s_{\beta}}\left(c_{1}, c_{2}\right), \psi, \psi^{\prime}\right)\right| & \ll_{\varepsilon}\left(m_{1}, c_{1}\right)\left(m_{2}, n_{2}, c_{2}\right)\left(c_{1}^{2}, c_{2}\right) c_{1}^{-1 / 2} c_{2}^{1 / 2}\left(c_{1} c_{2}\right)^{\varepsilon} & \text { if } c_{1} \mid c_{2}, \\
\left|\mathrm{Kl}\left(n_{w_{0}}\left(c_{1}, c_{2}\right), \psi, \psi^{\prime}\right)\right| & \ll_{\varepsilon}\left(m_{1} m_{2}, n_{1} n_{2}, c_{1} c_{2}\right)^{1 / 2}\left(c_{1}, c_{2}\right)^{1 / 2} c_{1}^{1 / 2} c_{2}^{3 / 4}\left(c_{1} c_{2}\right)^{\varepsilon} . &
\end{aligned}
$$

Moreover, the Kloosterman sum $\mathrm{Kl}\left(n_{w}\left(c_{1}, c_{2}\right), \psi, \psi^{\prime}\right)$ vanishes if the conditions on the right are not satisfied.

Theorems 1.1, 1.2 are proved in Sect. 4. We develop a stratification of $\operatorname{Sp}(2 r)$ Kloosterman sums in Sect. 2, generalising the stratification of GL( $r)$ Kloosterman sums introduced by Stevens [14]. Let

$$
\mathcal{T}:=\left\{\left(\begin{array}{c}
A \\
c A^{-1}
\end{array}\right) \in \operatorname{GL}\left(2 r, \mathbb{Z}_{p}\right) \mid A=\operatorname{diag}\left(a_{1}, a_{2}, \ldots, a_{r}\right), a_{1}, \ldots, a_{r}, c \in \mathbb{Z}_{p}^{\times}\right\} .
$$

be a subgroup of diagonal matrices. We will show that for $n \in N\left(\mathbb{Q}_{p}\right)$, there is a group action $\mathcal{T} \times X(n) \rightarrow X(n)$ sending $(t, x)$ to $t x s^{-1}$, where $s=n^{-1} t n$. The Kloosterman sum, as a sum over $X(n)$, can then be partitioned into sums over $\mathcal{T}$ orbits, in Theorem 2.4. The summands are then evaluated using results of Adolphson and Sperber [1] for multi-dimensional exponential sums of Laurent polynomials, as well as the $p$-adic stationary phase method for higher prime powers.

We give some brief comments on the bounds obtained here. As we shall see in Sect. 3, the Kloosterman sums corresponding $w=s_{\alpha}, s_{\beta}$ are just classical Kloosterman sums, and the bounds given here are just the optimal bounds for classical Kloosterman sums. The Kloosterman sums corresponding to $w=s_{\alpha} s_{\beta}, s_{\beta} s_{\alpha}$ can be expressed in terms of exponential sums of Laurent polynomials, whose optimal bounds are also well understood. Meanwhile, the bounds for $w=s_{\alpha} s_{\beta} s_{\alpha}, s_{\beta} s_{\alpha} s_{\beta}, w_{0}$, obtained using the stratification technique, are believed to be not optimal, since only the cancellations within individual $\mathcal{T}$-orbits are considered. In particular, we expect square-root cancellation for Kloosterman sums $\mathrm{Kl}_{p}\left(n_{w_{0}, r, s}, \psi_{m_{1}, m_{2}}, \psi_{n_{1}, n_{2}}\right)$ when $m_{1}, m_{2}, n_{1}, n_{2}$ are coprime to $p$, but this is beyond reach of current methods.

Let $F: T\left(\mathbb{R}^{+}\right) \rightarrow \mathbb{C}$ be a smooth function with rapid decay. Let $\psi, \psi^{\prime}$ be characters of $U(\mathbb{R})$ trivial on $U(\mathbb{Z})$. For $g=u y \in G / K$, where $u \in U(\mathbb{R}), y \in T\left(\mathbb{R}^{+}\right)$, define $\mathcal{F}_{\psi}(g):=\psi(\eta) F(y)$. The symplectic Poincaré series associated to $F$ is given by

$$
P_{\psi}(g)=\sum_{\gamma \in P_{0} \cap \Gamma \backslash \Gamma} \mathcal{F}_{\psi}(\gamma g)
$$


where $\Gamma=\operatorname{Sp}(2 r, \mathbb{Z})$, and $P_{0}$ is the standard minimal parabolic subgroup of $G$. The $\psi^{\prime}$-th Fourier coefficient of $P_{\psi}(g)$ is given by

$$
P_{\psi, \psi^{\prime}}(g)=\int_{U(\mathbb{Z}) \backslash U(\mathbb{R})} P_{\psi}(u g) \overline{\psi^{\prime}}(u) d u .
$$

We compute in Sect. 5 the Fourier coefficients $P_{\psi, \psi^{\prime}}(g)$ of the Poincaré series $P_{\psi}(g)$, in terms of auxiliary Kloosterman sums, which are also defined in Sect. 5. The bounds given in Theorem 1.1 also apply to these auxiliary Kloosterman sums, via Proposition 5.2.

\section{Stratification of symplectic Kloosterman sums}

Consider the subgroup of diagonal matrices

$$
\mathcal{T}:=\left\{\left(\begin{array}{c}
A \\
c A^{-1}
\end{array}\right) \in \operatorname{GL}\left(2 r, \mathbb{Z}_{p}\right) \mid A=\operatorname{diag}\left(a_{1}, a_{2}, \ldots, a_{r}\right), a_{1}, \ldots, a_{r}, c \in \mathbb{Z}_{p}^{\times}\right\} .
$$

Note that in general elements of $\mathcal{T}$ are not symplectic.

Lemma 2.1 Let $u \in U\left(\mathbb{Q}_{p}\right)$, and $t \in \mathcal{T}$. Then tut ${ }^{-1} \in U\left(\mathbb{Q}_{p}\right)$.

Proof Trivial.

Lemma 2.2 Let $n \in N\left(\mathbb{Q}_{p}\right)$, and $t \in \mathcal{T}$. Then $n^{-1}$ tn $\in \mathcal{T}$.

Proof Suppose $t=\operatorname{diag}\left(a_{1}, \ldots, a_{r}, c a_{1}^{-1}, \ldots, c a_{r}^{-1}\right)$. Then in general $n^{-1} t n$ has the form

$$
n^{-1} t n=\operatorname{diag}\left(\tau_{\sigma(1)}\left(a_{\sigma(1)}\right), \ldots, \tau_{\sigma(r)}\left(a_{\sigma(r)}\right), \tau_{\sigma(1)}\left(c a_{\sigma(1)}^{-1}\right), \ldots, \tau_{\sigma(r)}\left(c a_{\sigma(r)}^{-1}\right)\right),
$$

where $\sigma$ is a permutation of $\{1, \ldots, r\}$, and $\tau_{i}:\left\{a_{i}, c a_{i}^{-1}\right\} \rightarrow\left\{a_{i}, c a_{i}^{-1}\right\}$ are permutations for $i=1, \ldots, r$. Since $a_{i}=c\left(c a_{i}^{-1}\right)^{-1}$, we see that

$$
n^{-1} t n=\operatorname{diag}\left(\tau_{\sigma(1)}\left(a_{\sigma(1)}\right), \ldots, \tau_{\sigma(r)}\left(a_{\sigma(r)}\right), c\left(\tau_{\sigma(1)}\left(a_{\sigma(1)}\right)\right)^{-1}, \ldots, c\left(\tau_{\sigma(r)}\left(a_{\sigma(r)}\right)\right)^{-1}\right) \in \mathcal{T} .
$$

Let $x=u n u^{\prime} \in C(n)$, and $t \in \mathcal{T}$. By Lemma 2.2, $s:=n^{-1} t n \in \mathcal{T}$. By Lemma 2.1, we see that

$$
t * x:=t x s^{-1}=\left(t u t^{-1}\right) n\left(s u^{\prime} s^{-1}\right) \in U\left(\mathbb{Q}_{p}\right) n U\left(\mathbb{Q}_{p}\right) \cap G\left(\mathbb{Z}_{p}\right)=C(n) .
$$

As conjugation by $t$ and $s$ preserves $U\left(\mathbb{Z}_{p}\right)$ and $U_{n}\left(\mathbb{Z}_{p}\right)$, this induces an action on $X(n)=U\left(\mathbb{Z}_{p}\right) \backslash C(n) / U_{n}\left(\mathbb{Z}_{p}\right):$

$$
\mathcal{T} \times X(n) \rightarrow X(n), \quad(t, x) \mapsto t * x .
$$


For characters $\psi: U\left(\mathbb{Q}_{p}\right) / U\left(\mathbb{Z}_{p}\right) \rightarrow \mathbb{C}^{\times}, \psi^{\prime}: U_{n}\left(\mathbb{Q}_{p}\right) / U_{n}\left(\mathbb{Z}_{p}\right) \rightarrow \mathbb{C}^{\times}$, decomposition of $X(n)$ into $\mathcal{T}$-orbits gives a decomposition of Kloosterman sums:

$$
\mathrm{Kl}_{p}(n, \psi, \psi)=\sum_{x \in \mathcal{T} \backslash X(n)} \sum_{y \in \mathcal{T} * x} \psi(u(y)) \psi^{\prime}\left(u^{\prime}(y)\right) .
$$

Let $\alpha_{i}=e_{i}-e_{i+1}, 1 \leq i \leq r-1$, and $\alpha_{r}=2 e_{r}$ be the simple roots of $T$ in $G$. Denote $\Delta=\left\{\alpha_{1}, \ldots, \alpha_{r}\right\}$, and $\Delta_{w}=\{\alpha \in \Delta \mid w(\alpha)<0\}$. Let $U_{\alpha_{i}}\left(\mathbb{Q}_{p}\right) \subseteq U\left(\mathbb{Q}_{p}\right)$ be the root subgroup corresponding to $\alpha_{i}$. For $u \in U\left(\mathbb{Q}_{p}\right)$, we also use $\alpha_{i}$ to denote the canonical projection map

$$
\alpha_{i}: U\left(\mathbb{Q}_{p}\right) \rightarrow U_{\alpha_{i}}\left(\mathbb{Q}_{p}\right) \simeq \mathbb{Q}_{p}
$$

Explicitly, for $u=\left(u_{i j}\right) \in U\left(\mathbb{Q}_{p}\right)$, the projection maps are given by

$$
\begin{aligned}
& \alpha_{i}(u)=u_{i, i+1}, \quad 1 \leq i \leq r-1, \\
& \alpha_{r}(u)=u_{r, 2 r}
\end{aligned}
$$

Characters of $U\left(\mathbb{Q}_{p}\right) / U\left(\mathbb{Z}_{p}\right)$ have the form

$$
\psi(u)=\psi_{n_{1}, \ldots, n_{r}}(u):=\prod_{i=1}^{r} \mathrm{e}\left(n_{i} \alpha_{i}(u)\right), \quad n_{i} \in \mathbb{Z},
$$

where e $: \mathbb{Q}_{p} / \mathbb{Z}_{p} \rightarrow \mathbb{C}^{\times}$is the standard additive character. For $x=u(x) n u^{\prime}(x)$, define projections

$$
\kappa_{i}(x):=\alpha_{i}(u(x)), \quad \kappa_{i}^{\prime}(x):=\alpha_{i}\left(u^{\prime}(x)\right), \quad 1 \leq i \leq r .
$$

Note that $\kappa_{i}^{\prime}(x)=0$ unless $\alpha_{i} \in \Delta_{w}(n)$. For $u \in U\left(\mathbb{Q}_{p}\right)$, and $t=$ $\operatorname{diag}\left(a_{1}, \ldots, a_{r}, c a_{1}^{-1}, \ldots, c a_{r}^{-1}\right) \in \mathcal{T}$, we compute that

$$
\begin{aligned}
& \alpha_{i}\left(t u t^{-1}\right)=a_{i} a_{i+1}^{-1} \alpha_{i}(u), \quad 1 \leq i \leq r-1, \\
& \alpha_{r}\left(t u t^{-1}\right)=c^{-1} a_{r}^{2} \alpha_{r}(u) .
\end{aligned}
$$

Suppose $t=\operatorname{diag}\left(a_{1}, \ldots, a_{r}, c a_{1}^{-1}, \ldots, c a_{r}^{-1}\right) \in \mathcal{T}$, and $s=n^{-1}$ tn $=$ $\operatorname{diag}\left(a_{1}^{\prime}, \ldots, a_{r}^{\prime}, c a_{1}^{\prime-1}, \ldots c a_{r}^{\prime-1}\right) \in \mathcal{T}$. Note from the proof of Lemma 2.2 that we have the same $c$. Then we have

$$
\begin{aligned}
& \kappa_{i}(t * x)=a_{i} a_{i+1}^{-1} \kappa_{i}(x), \quad 1 \leq i \leq r-1, \\
& \kappa_{r}(t * x)=c^{-1} a_{r}^{2} \kappa_{r}(x),
\end{aligned}
$$


and

$$
\begin{aligned}
\kappa_{i}^{\prime}(t * x) & =a_{i}^{\prime} a_{i+1}^{\prime}{ }^{-1} \kappa_{i}^{\prime}(x), \quad 1 \leq i \leq r-1, \\
\kappa_{r}^{\prime}(t * x) & =c^{-1} a_{r}^{\prime 2} \kappa_{r}^{\prime}(x) .
\end{aligned}
$$

For $\ell \in \mathbb{N}$, we define

$$
A_{w}(\ell):=\left(\mathbb{Z} / p^{\ell} \mathbb{Z}\right)^{\Delta} \times\left(\mathbb{Z} / p^{\ell} \mathbb{Z}\right)^{\Delta_{w}}
$$

and

$$
V_{w}(\ell):=\left\{\left(\lambda, \lambda^{\prime}\right) \in A_{w}(\ell)^{\times} \mid \begin{array}{l}
\exists t \in \mathcal{T} \text { such that } \kappa_{i}(t * x)=\lambda_{i} \kappa_{i}(x), \kappa_{j}^{\prime}(t * x)=\lambda_{j}^{\prime} \kappa_{j}^{\prime}(x) \\
\text { for } x \in X(n), 1 \leq i, j \leq r, \alpha_{j} \in \Delta_{w}
\end{array}\right\} .
$$

Lemma 2.3 We have $\left|V_{w}(\ell)\right|=\left(p^{\ell}\left(1-p^{-1}\right)\right)^{r}$.

Proof For every $\lambda \in\left(\left(\mathbb{Z} / p^{\ell} \mathbb{Z}\right)^{\times}\right)^{\Delta}$, we can find $t \in \mathcal{T}$ such that $\kappa_{i}(t * x)=\lambda_{i} \kappa_{i}(x)$ for $1 \leq i \leq r$. Using (2.2), we find a unique $\lambda^{\prime} \in\left(\left(\mathbb{Z} / p^{\ell} \mathbb{Z}\right)^{\times}\right)^{\Delta_{w}}$ such that $\kappa_{i}^{\prime}(t * x)=$ $\lambda_{i}^{\prime} \kappa_{i}(x)$ for $1 \leq j \leq r$ with $\alpha_{j} \in \Delta_{w}$, and it is straightforward to check that $\lambda^{\prime}$ is independent of the choice of $t \in \mathcal{T}$. Therefore

$$
\left|V_{w}(\ell)\right|=\left|\left(\left(\mathbb{Z} / p^{\ell} \mathbb{Z}\right)^{\times}\right)^{\Delta}\right|=\left(p^{\ell}\left(1-p^{-1}\right)\right)^{r}
$$

as claimed.

For a character $\theta: A_{w}(\ell) \rightarrow \mathbb{C}^{\times}$, we define

$$
S_{w}(\theta ; \ell)=\sum_{v \in V_{w}(\ell)} \theta(v)
$$

Theorem 2.4 Let $n \in N\left(\mathbb{Q}_{p}\right)$, and suppose $\ell$ is large enough such that the matrix entries of $u(x), u^{\prime}(x)$ lie in $p^{-\ell} \mathbb{Z}_{p} / \mathbb{Z}_{p}$ for every $x \in X(n)$. Let $\psi=\psi_{n_{1}, \ldots, n_{r}}$ : $U\left(\mathbb{Q}_{p}\right) / U\left(\mathbb{Z}_{p}\right) \rightarrow \mathbb{C}^{\times}$and $\psi^{\prime}=\left.\psi_{n_{1}^{\prime}, \ldots, n_{r}^{\prime}}\right|_{U_{n}\left(\mathbb{Q}_{p}\right)}: U_{n}\left(\mathbb{Q}_{p}\right) / U_{n}\left(\mathbb{Z}_{p}\right) \rightarrow \mathbb{C}^{\times}$be characters. Define the character $\theta_{x}: A_{w}(\ell) \rightarrow \mathbb{C}^{\times}$by

$$
\theta_{x}\left(\lambda, \lambda^{\prime}\right)=\prod_{i=1}^{r} \mathrm{e}\left(\lambda_{i} n_{i} \kappa_{i}(x)\right) \prod_{\substack{i=1 \\ w\left(\alpha_{i}\right)<0}}^{r} \mathrm{e}\left(\lambda_{i}^{\prime} n_{i}^{\prime} \kappa_{i}^{\prime}(x)\right)
$$

Then

$$
\mathrm{Kl}_{p}\left(n, \psi, \psi^{\prime}\right)=\left(p^{\ell}\left(1-p^{-1}\right)\right)^{-r} \sum_{x \in \mathcal{T} \backslash X(n)} \mathfrak{N}(x) S_{w}\left(\theta_{x} ; \ell\right),
$$


where $\mathfrak{N}(x)=|\mathcal{T} * x|$ is the size of $\mathcal{T}$-orbit of $x \in X(n)$.

Proof We rewrite the Kloosterman sum

$$
\begin{aligned}
\mathrm{K}_{p}\left(n, \psi, \psi^{\prime}\right) & =\sum_{x \in \mathcal{T} \backslash X(n)} \sum_{y \in \mathcal{T} * x} \psi(u(y)) \psi^{\prime}\left(u^{\prime}(y)\right) \\
& =\sum_{x \in \mathcal{T} \backslash X(n)} \sum_{y \in \mathcal{T} * x} \prod_{i=1}^{r} \mathrm{e}\left(n_{i} \kappa_{i}(y)\right) \prod_{\substack{i=1 \\
w\left(\alpha_{i}\right)<0}}^{r} \mathrm{e}\left(n_{i}^{\prime} \kappa_{i}^{\prime}(y)\right) .
\end{aligned}
$$

For $\left(\lambda, \lambda^{\prime}\right) \in V_{w}(\ell)$, we can find $t \in \mathcal{T}$ such that $\kappa_{i}(t * x)=\lambda_{i} \kappa_{i}(x), \kappa_{j}^{\prime}(t * x)=$ $\lambda_{i}^{\prime} \kappa_{j}^{\prime}(x)$ for $x \in X(n), 1 \leq i, j \leq r, w\left(\alpha_{j}\right)<0$. Hence

$$
\begin{gathered}
\sum_{y \in \mathcal{T} * x} \prod_{i=1}^{r} \mathrm{e}\left(\lambda_{i} n_{i} \kappa_{i}(y)\right) \prod_{\substack{i=1 \\
w\left(\alpha_{i}\right)<0}}^{r} \mathrm{e}\left(\lambda_{i}^{\prime} n_{i}^{\prime} \kappa_{i}^{\prime}(y)\right) \\
=\sum_{y \in \mathcal{T} * x} \prod_{i=1}^{r} \mathrm{e}\left(n_{i} \kappa_{i}(t * y)\right) \prod_{\substack{i=1 \\
w\left(\alpha_{i}\right)<0}}^{r} \mathrm{e}\left(n_{i}^{\prime} \kappa_{i}^{\prime}(t * y)\right) \\
=\sum_{y \in \mathcal{T} * x} \prod_{i=1}^{r} \mathrm{e}\left(n_{i} \kappa_{i}(y)\right) \prod_{\substack{i=1 \\
w\left(\alpha_{i}\right)<0}}^{r} \mathrm{e}\left(n_{i}^{\prime} \kappa_{i}^{\prime}(y)\right) .
\end{gathered}
$$

Summing over $V_{w}(\ell)$, we have

$$
\begin{aligned}
& \left|V_{w}(\ell)\right| \mathrm{Kl}_{p}\left(n, \psi, \psi^{\prime}\right) \\
& \quad=\sum_{x \in \mathcal{T} \backslash X(n)} \sum_{y \in \mathcal{T} * x} \sum_{\left(\lambda, \lambda^{\prime}\right) \in V_{w}(\ell)} \prod_{i=1}^{r} \mathrm{e}\left(\lambda_{i} n_{i} \kappa_{i}(y)\right) \prod_{\substack{i=1 \\
w\left(\alpha_{i}\right)<0}}^{r} \mathrm{e}\left(\lambda_{i}^{\prime} n_{i}^{\prime} \kappa_{i}^{\prime}(y)\right) \\
& \quad=\sum_{x \in \mathcal{T} \backslash X(n)} \mathfrak{N}(x) \sum_{\left(\lambda, \lambda^{\prime}\right) \in V_{w}(\ell)} \prod_{i=1}^{r} \mathrm{e}\left(\lambda_{i} n_{i} \kappa_{i}(y)\right) \prod_{\substack{i=1 \\
w\left(\alpha_{i}\right)<0}}^{r} \mathrm{e}\left(\lambda_{i}^{\prime} n_{i}^{\prime} \kappa_{i}^{\prime}(y)\right) \\
& \quad=\sum_{x \in \mathcal{T} \backslash X(n)} \mathfrak{N}(x) S_{w}\left(\theta_{x} ; \ell\right) .
\end{aligned}
$$

Dividing both sides by $\left|V_{w}(\ell)\right|$ yields the statement.

\section{$3 \mathrm{Sp}(4)$ Kloosterman sums}

Now we give explicit formulations for Kloosterman sums for $G=\operatorname{Sp}\left(4, \mathbb{Q}_{p}\right)$, classified by the image $w(n)$ of the projection onto $W$. Fix $\psi=\psi_{m_{1}, m_{2}}, \psi^{\prime}=\psi_{n_{1}, n_{2}}$, with $\psi_{m_{1}, m_{2}}, \psi_{n_{1}, n_{2}}$ as in (1.1). 
Proposition 3.1 $\left[14\right.$, Theorem 3.2] Let $n \in N\left(\mathbb{Q}_{p}\right)$, and $\psi: U\left(\mathbb{Q}_{p}\right) / U\left(\mathbb{Z}_{p}\right) \rightarrow \mathbb{C}^{\times}$, $\psi^{\prime}: U_{n}\left(\mathbb{Q}_{p}\right) / U_{n}\left(\mathbb{Z}_{p}\right) \rightarrow \mathbb{C}^{\times}$be characters. If $t \in T\left(\mathbb{Z}_{p}^{\times}\right)$, then

$$
\begin{aligned}
\mathrm{K}_{p}\left(t n, \psi, \psi^{\prime}\right) & =\mathrm{K}_{p}\left(n, \psi_{t}, \psi^{\prime}\right), \\
\mathrm{Kl}_{p}\left(n t^{-1}, \psi, \psi^{\prime}\right) & =\mathrm{K}_{p}\left(n, \psi, \psi_{t}^{\prime}\right)
\end{aligned}
$$

where $\psi_{t}(u)=\psi\left(t u t^{-1}\right)$.

By Proposition 3.1, it suffices to consider Kloosterman sums $\mathrm{Kl}_{p}\left(n, \psi, \psi^{\prime}\right)$ for $n$ such that entries of $n$ are powers of $p$, and $X(n)$ is nonempty. To express the Kloosterman sums, we express the coset representatives for $X(n)$ in terms of Plücker coordinates, which were introduced in $[3,12]$. For $g=\left(g_{i j}\right) \in G=\operatorname{Sp}\left(4, \mathbb{Q}_{p}\right)$, we define Plücker coordinates

$$
\begin{array}{rlrl}
v_{i} & :=g_{3, i}, & 1 \leq i \leq 4, \\
v_{i j}:=g_{3, i} g_{4, j}-g_{3, j} g_{4, i}, & 1 \leq i<j \leq 4 .
\end{array}
$$

The Plücker coordinates satisfy the following relations:

$$
\begin{aligned}
v_{i} v_{j k}-v_{j} v_{i k}+v_{k} v_{i j} & =0, \quad 1 \leq i<j<k \leq 4, \\
v_{13}+v_{24} & =0 .
\end{aligned}
$$

Hence, we can associate to every $g \in G\left(\mathbb{Q}_{p}\right)$ its Plücker coordinates

$$
v=v_{g}=\left(v_{1}, v_{2}, v_{3}, v_{4} ; v_{12}, v_{13}, v_{14}, v_{23}, v_{24}, v_{34}\right) \in \mathbb{Q}_{p}^{10} .
$$

It follows from the definition that if $g \in G\left(\mathbb{Z}_{p}\right)$, then the corresponding Plücker coordinates $v_{g}$ are integral, and satisfy the coprimality conditions

$$
\left(v_{1}, v_{2}, v_{3}, v_{4}\right)=1, \quad\left(v_{12}, v_{13}, v_{14}, v_{23}, v_{24}, v_{34}\right)=1
$$

It is proved in [12] that there is a bijection

$$
\left\{\operatorname{cosets} U\left(\mathbb{Q}_{p}\right) \backslash G\left(\mathbb{Z}_{p}\right)\right\} \longleftrightarrow\left\{v_{g} \in \mathbb{Z}_{p}^{10} \mid v_{g}\right. \text { satisfies (3.1) and (3.2)\} . }
$$

In particular, this means the coset representatives for $X(n)$ can be described using Plücker coordinates. For notational convenience, for $n \in N\left(\mathbb{Q}_{p}\right)$ we write $X^{v}(n)$ for a complete system of coset representatives of $X(n)$, in terms of Plücker coordinates.

Now we give explicit formulations for Kloosterman sums $\mathrm{Kl}_{p}\left(n, \psi, \psi^{\prime}\right)$. Note that by Proposition 3.1, it suffices to consider the case $n=n_{w, r, s}$, where $n_{w, r, s}$ is given as in (1.2). By looking at the Plücker coordinates, one deduces that $X\left(n_{w, r, s}\right)$ is nonempty only if $r, s \geq 0$. Explicit formulations for $X^{v}\left(n_{w, r, s}\right)$ are obtained by unfolding the conditions (3.1) and (3.2), and are given in [12], and we shall use the results from there directly. 
(i) $n=n_{\mathrm{id}, r, s}$. Then $X\left(n_{\mathrm{id}, r, s}\right)$ is empty unless $r=s=0$, where $X\left(n_{\mathrm{id}, 0,0}\right)=\left\{I_{4}\right\}$ is a singleton. So the Kloosterman sum is trivial:

$$
\mathrm{Kl}_{p}\left(n_{\mathrm{id}, 0,0}, \psi, \psi^{\prime}\right)=1
$$

(ii) $n=n_{s_{\alpha}, r, s}$. Then $X\left(n_{s_{\alpha}, r, s}\right)$ is nonempty when $s=0$. In this case we have

$$
X^{v}\left(n_{s_{\alpha}, r, 0}\right)=\left\{\left(0,0, v_{3}, p^{r} ; 0,0,0,0,0,1\right)\right\},
$$

where $v_{3}\left(\bmod p^{r}\right)$ such that $\left(v_{3}, p^{r}\right)=1$. The corresponding Kloosterman sum is actually a GL(2) Kloosterman sum:

$$
\mathrm{Kl}_{p}\left(n_{s_{\alpha}, r, 0}, \psi, \psi^{\prime}\right)=S\left(m_{1}, n_{1} ; p^{r}\right)
$$

(iii) $n=n_{s_{\beta}, r, s}$. Then $X\left(n_{s_{\beta}, r, s}\right)$ is nonempty when $r=0$. In this case we have

$$
X^{v}\left(n_{s_{\beta}, 0, s}\right)=\left\{\left(0,0,1,0 ; 0,0,0, p^{s}, 0, v_{34}\right)\right\}
$$

where $v_{34}\left(\bmod p^{s}\right)$ such that $\left(v_{34}, p^{s}\right)=1$. The corresponding Kloosterman sum is actually a GL(2) Kloosterman sum:

$$
\mathrm{Kl}_{p}\left(n_{s_{\beta}, 0, s}, \psi, \psi^{\prime}\right)=S\left(m_{2}, n_{2} ; p^{s}\right)
$$

(iv) $n=n_{s_{\alpha} s_{\beta}, r, s}$. Then $X\left(n_{s_{\alpha} s_{\beta}, r, s}\right)$ is nonempty when $r \geq s$. Unfolding the conditions (3.1) and (3.2), we compute

$$
X^{v}\left(n_{s_{\alpha} s_{\beta}, r, s}\right)=\left\{\left(0, p^{r}, v_{3}, v_{4} ; 0,0,0, p^{s}, 0,-v_{4} p^{s-r}\right)\right\},
$$

where $v_{3}, v_{4}\left(\bmod p^{r}\right)$ such that $\left(v_{4}, p^{r}\right)=p^{r-s}$ and $\left(v_{3}, p^{r-s}\right)=1$. We write $v_{4}=v_{4}^{\prime} p^{r-s}$, so $\left(v_{4}^{\prime}, p^{s}\right)=1$. Bruhat decomposition gives

$$
\begin{aligned}
x & =\left(\begin{array}{cccc}
1 & \beta_{1} & \beta_{2} & \beta_{3} \\
& 1 & \beta_{4} & \beta_{5} \\
& & 1 & \\
& & -\beta_{1} & 1
\end{array}\right)\left(\begin{array}{ccc}
p^{r-s} & & \\
& p^{r} & \\
& & p^{s-r}
\end{array}\right)\left(\begin{array}{ccc}
1 & \\
1 & v_{3} p^{-r} & v_{3}^{\prime} p^{-s} \\
& 1 & \\
& & 1
\end{array}\right) \\
& =\left(\begin{array}{cccc}
\beta_{1} p^{r-s} & \beta_{2} p^{r} & \beta_{2} v_{3}+\beta_{3} p^{s-r} & \beta_{2} v_{4}^{\prime} p^{r-s}+\beta_{1} v_{3} p^{-s}-p^{-r} \\
p^{r-s} & \beta_{4} p^{r} & \beta_{4} v_{3}+\beta_{5} p^{s-r} & \beta_{4} v_{4}^{\prime} p^{r-s}+v_{3} p^{-s} \\
0 & p^{r} & v_{3} & v_{4}^{\prime} p^{r-s} \\
0 & -\beta_{1} p^{r} & -\beta_{1} v_{3}+p^{s-r} & -\beta_{1} v_{4}^{\prime} p^{r-s}
\end{array}\right) .
\end{aligned}
$$

The entry $-\beta_{1} v_{3}+p^{s-r}$ being an integer says $\beta_{1} \equiv \overline{v_{3}} p^{s-r}(\bmod 1)$. The entry $\beta_{4} v_{4}^{\prime} p^{r-s}+v_{3} p^{-s}$ being an integer says $\beta_{4} \equiv-\overline{v_{4}^{\prime}} v_{3} p^{-r}\left(\bmod p^{s-r}\right)$. Write $\beta_{4}=-\overline{v_{4}^{\prime}} v_{3} p^{-r}+\gamma_{4} p^{s-r}$ for some $\gamma_{4} \in \mathbb{Z}$. The entry $\beta_{4} v_{3}+\beta_{5} p^{s-r}$ being an 
integer says $\gamma_{4} v_{3}+\beta_{5} \equiv \overline{v_{4}^{\prime}} v_{3}^{2} p^{-s}\left(\bmod p^{r-s}\right)$, hence $\beta_{5} \equiv \overline{v_{4}^{\prime}} v_{3}^{2} p^{-s}(\bmod 1)$. After writing $v_{4}$ for $v_{4}^{\prime}$, the Kloosterman sum is given by

$$
\mathrm{K}_{p}\left(n_{s_{\alpha} s_{\beta}, r, s}, \psi, \psi^{\prime}\right)=\sum_{\substack{v_{4}\left(\bmod p^{s}\right) \\\left(v_{4}, p^{s}\right)=1}} \sum_{\substack{v_{3}\left(\bmod p^{r}\right) \\\left(v_{3}, p^{r-s}\right)=1}} \mathrm{e}\left(\frac{m_{1} \overline{v_{3}} p^{s}}{p^{r}}\right) \text { e }\left(\frac{m_{2} \overline{v_{4}} v_{3}^{2}+n_{2} v_{4}}{p^{s}}\right) .
$$

(v) $n=n_{s_{\beta} s_{\alpha}, r, s}$. Then $X\left(n_{s_{\beta} s_{\alpha}, r, s}\right)$ is nonempty when $s \geq 2 r$. Unfolding the conditions (3.1) and (3.2), we compute

$$
X^{v}\left(n_{s_{\beta} s_{\alpha}, r, s}\right)=\left\{\left(0,0,-v_{24} p^{r-s}, p^{r} ; 0,-v_{24}, p^{s},-v_{24} p^{-s}, v_{24}, v_{34}\right)\right\}
$$

where $v_{24}, v_{34}\left(\bmod p^{s}\right)$ such that $\left(v_{24}, p^{s}\right)=p^{s-r}$ and $\left(v_{34}, p^{s-2 r}\right)=1$. We write $v_{24}=v_{24}^{\prime} p^{s-r}$, so $\left(v_{24}^{\prime}, p^{r}\right)=1$. Bruhat decomposition gives

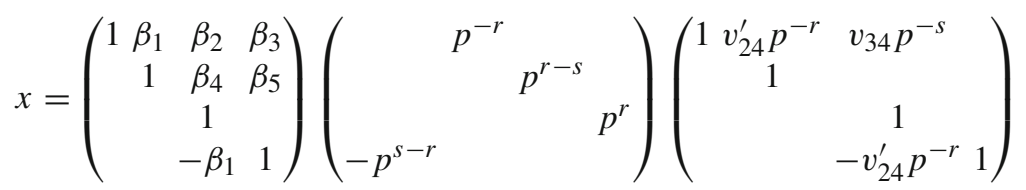

$$
\begin{aligned}
& =\left(\begin{array}{cccc}
-\beta_{3} p^{s-r} & -\beta_{3} v_{24}^{\prime} p^{s-2 r}+p^{-r} & -\beta_{2} v_{24}^{\prime}-\beta_{3} v_{34} p^{-r}+\beta_{1} p^{r-s} & \beta_{2} p^{r} \\
-\beta_{5} p^{s-r} & -\beta_{5} v_{24}^{\prime} p^{s-2 r} & -\beta_{4} v_{24}^{\prime}-\beta_{5} v_{34} p^{-r}+p^{r-s} & \beta_{4} p^{r} \\
0 & 0 & -v_{24}^{\prime} & p^{r} \\
-p^{s-r} & -v_{24}^{\prime} p^{s-2 r} & \beta_{1} v_{24}^{\prime}-v_{34} p^{-r} & -\beta_{1} p^{r}
\end{array}\right) .
\end{aligned}
$$

The entry $\beta_{1} v_{24}^{\prime}-v_{34} p^{-r}$ being an integer says $\beta_{1} \equiv \overline{v_{24}^{\prime}} v_{34} p^{-r}(\bmod 1)$. The entry $\beta_{4} p^{r}$ being an integer says $\beta_{4}=\beta_{4}^{\prime} p^{-r}$ for some $\beta_{4}^{\prime} \in \mathbb{Z}$. The entry $-\beta_{4} v_{24}^{\prime}$ $\beta_{5} v_{34} p^{-r}+p^{r-s}$ being an integer says $\beta_{4}^{\prime} v_{24}^{\prime}+\beta_{5} v_{34} \equiv p^{2 r-s}\left(\bmod p^{r}\right)$, hence $\beta_{5} \equiv \overline{v_{34}} p^{2 r-s}(\bmod 1)$. After writing $v_{24}$ for $v_{24}^{\prime}$, the Kloosterman sum is given by

$$
\begin{aligned}
\mathrm{K}_{p}\left(n_{s_{\beta} s_{\alpha}, r, s}, \psi, \psi^{\prime}\right) & \sum_{\substack{v_{24}\left(\bmod p^{r}\right) \\
\left(v_{24}, p^{r}\right)=1}} \sum_{\substack{v_{34}\left(\bmod p^{s}\right) \\
\left(v_{34}, p^{s-2 r}\right)=1}} \mathrm{e}\left(\frac{m_{1} \overline{v_{24}} v_{34}+n_{1} v_{24}}{p^{r}}\right) \mathrm{e}\left(\frac{m_{2} \overline{v_{34}} p^{2 r}}{p^{s}}\right) .
\end{aligned}
$$

Remark. This Kloosterman sum is related to a GL(3) Kloosterman sum. Precisely, following the notation in $[2,(4.3)]$, we have

$$
\mathrm{Kl}_{p}\left(n_{s_{\beta} s_{\alpha}, r, s}, \psi, \psi^{\prime}\right)=p^{r} S\left(n_{1}, m_{1}, m_{2} ; p^{r}, p^{s-r}\right) .
$$

A non-trivial bound for $\mathrm{Kl}_{p}\left(n_{s_{\beta} s_{\alpha}, r, s}, \psi, \psi^{\prime}\right)$ then follows from Larsen [2, Appendix]. 


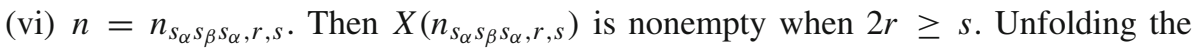
conditions (3.1) and (3.2), we compute

$$
\begin{aligned}
& X\left(n_{s_{\alpha} s_{\beta} s_{\alpha}, r, s}\right) \\
& \quad=\left\{\left(p^{r}, v_{2}, v_{3}, v_{4} ; 0,-v_{2} p^{s-r}, p^{s},-v_{2}^{2} p^{s-2 r}, v_{2} p^{s-r},\left(p^{r} v_{3}+v_{2} v_{4}\right) p^{s-2 r}\right)\right\},
\end{aligned}
$$

where $v_{2}, v_{3}, v_{4}\left(\bmod p^{r}\right)$, such that $\left(v_{2}, v_{3}, v_{4}, p^{r}\right)=1$, and if $d:=\left(v_{2}, p^{r}\right)$, then $\left(d^{2}, p^{r} v_{3}+v_{2} v_{4}\right)=p^{2 r-s}$. Let $d=p^{r-a}$. Then $a$ satisfies $s-r \leq a \leq s / 2$. We write $v_{2}=v_{2}^{\prime} p^{r-a}$, so $\left(v_{2}^{\prime}, p^{a}\right)=1$. Bruhat decomposition gives



$$
\begin{aligned}
& =\left(\begin{array}{cccc}
\beta_{2} p^{r} & \beta_{2} v_{2}^{\prime} p^{r-a}+\beta_{1} p^{r-s} & \beta_{2} v_{3}-\beta_{3} v_{2}^{\prime} p^{s-a-r}+\beta_{1} v_{4} p^{-s}-p^{-r} & \beta_{2} v_{4}+\beta_{3} p^{s-r} \\
\beta_{4} p^{r} & \beta_{4} v_{2}^{\prime} p^{r-a}+p^{r-s} & \beta_{4} v_{3}-\beta_{5} v_{2}^{\prime} p^{s-a-r}+v_{4} p^{-s} & \beta_{4} v_{4}+\beta_{5} p^{s-r} \\
p^{r} & v_{2}^{\prime} p^{r-a} & v_{3} & v_{4} \\
-\beta_{1} p^{r} & -\beta_{1} v_{2}^{\prime} p^{r-a} & -\beta_{1} v_{3}-v_{2}^{\prime} p^{s-a-r} & -\beta_{1} v_{4}+p^{s-r}
\end{array}\right) .
\end{aligned}
$$

The entry $-\beta_{1} v_{2}^{\prime} p^{r-a}$ being an integer says $\beta_{1}=\beta_{1}^{\prime} p^{a-r}$ for some $\beta_{1}^{\prime} \in \mathbb{Z}$. Entries $-\beta_{1} v_{3}-v_{2}^{\prime} p^{s-a-r}$ and $-\beta_{1} v_{4}+p^{s-r}$ being integers says

$$
\beta_{1}^{\prime} v_{3} \equiv-v_{2}^{\prime} p^{s-2 a} \quad\left(\bmod p^{r-a}\right), \quad \beta_{1}^{\prime} v_{4} \equiv p^{s-a} \quad\left(\bmod p^{r-a}\right)
$$

As $\left(v_{3}, v_{4}, p^{r-a}\right)=1$, these equations determine $\beta_{1}$ uniquely modulo 1 .

The entry $\beta_{4} v_{2}^{\prime} p^{r-a}+p^{r-s}$ being an integer says $\beta_{4} \equiv-\overline{v_{2}^{\prime}} p^{a-s}\left(\bmod p^{a-r}\right)$. Write $\beta_{4}=-\overline{v_{2}^{\prime}} p^{a-s}+\gamma_{4} p^{a-r}$ for some $\gamma_{4} \in \mathbb{Z}$. Then $\beta_{4} v_{3}-\beta_{5} v_{2}^{\prime} p^{s-a-r}+v_{4} p^{-s}$ being an integer says

$$
-\overline{v_{2}^{\prime}} v_{3} p^{a}+\gamma_{4} v_{3} p^{s+a-r}-\beta_{5} v_{2}^{\prime} p^{2 s-a-r}+v_{4} \equiv 0 \quad\left(\bmod p^{s}\right) .
$$

Write $\beta_{5}=\beta_{5}^{\prime} p^{a+r-2 s}$ for some $\beta_{5}^{\prime} \in \mathbb{Z}$. Then we solve

$$
\beta_{5}^{\prime} \equiv-{\overline{v_{2}^{\prime}}}^{2} v_{3} p^{a}+\gamma_{4} \overline{v_{2}^{\prime}} v_{3} p^{s+a-r}+\overline{v_{2}^{\prime}} v_{4} \quad\left(\bmod p^{s}\right)
$$

Then $\beta_{4} v_{4}+\beta_{5} p^{s-r}$ being an integer says

$$
\gamma_{4}\left(p^{a} v_{3}+v_{2}^{\prime} v_{4}\right) p^{s+a-r} \equiv v_{3} p^{2 a} \quad\left(\bmod p^{s}\right) .
$$

Recall that $\left(p^{r-a}, p^{a} v_{3}+v_{2}^{\prime} v_{4}\right)=p^{r+a-s}$. Hence, unless $a=\frac{s}{2}$, we can write $p^{a} v_{3}+v_{2}^{\prime} v_{4}=V^{\prime} p^{r+a-s}$, with $\left(V^{\prime}, p\right)=1$. Then we solve (3.6):

$$
\gamma_{4} \equiv \overline{V^{\prime}} v_{3} \quad\left(\bmod p^{s-2 a}\right)
$$


Putting back to (3.5) gives

$$
\beta_{5}^{\prime} \equiv-{\overline{v_{2}^{\prime}}}^{2} v_{3} p^{a}+\overline{V^{\prime} v_{2}^{\prime}} v_{3}^{2} p^{s+a-r}+\overline{v_{2}^{\prime}} v_{4} \quad\left(\bmod p^{2 s-a-r}\right),
$$

hence $\beta_{5}$ is uniquely determined modulo 1 . When $a=\frac{s}{2}, \gamma_{4}$ can be arbitrary, and we have

$$
\beta_{5}^{\prime} \equiv-{\overline{v_{2}^{\prime}}}^{2} v_{3} p^{a}+{\overline{v_{2}^{\prime}}}_{4} \quad\left(\bmod p^{2 s-a-r}\right)
$$

hence $\beta_{5}$ is also uniquely determined modulo 1 in this case.

So, after writing $u$ for $\beta_{5} p^{s}$, the Kloosterman sum is given by

$$
\begin{gathered}
\mathrm{Kl}_{p}\left(n_{s_{\alpha} s_{\beta} s_{\alpha}, r, s}, \psi, \psi^{\prime}\right) \\
=\sum_{s-r \leq a \leq s / 2} \sum_{\begin{array}{c}
v_{2}, v_{3}, v_{4}\left(\bmod p^{r}\right) \\
v_{2}=v_{2}^{\prime} p^{r-a},\left(v_{2}^{\prime}, p^{a}\right)=1 \\
\left(v_{3}, v_{4}, p^{r-a}\right)=1 \\
\left(p^{r-a}, p^{a} v_{3}+v_{2}^{\prime} v_{4}\right)=p^{r+a-s}
\end{array}} \mathrm{e}\left(\frac{m_{1} \hat{v}_{2}+n_{1} v_{2}}{p^{r}}\right) \mathrm{e}\left(\frac{m_{2} u}{p^{s}}\right),
\end{gathered}
$$

where $\hat{v}_{2}$ is chosen modulo $p^{r}$ such that

$$
\hat{v}_{2} v_{3} \equiv-v_{2}^{\prime} p^{s-a} \quad\left(\bmod p^{r}\right), \quad \hat{v}_{2} v_{4} \equiv p^{s} \quad\left(\bmod p^{r}\right)
$$

and

$$
u \equiv \begin{cases}-{\overline{v_{2}^{\prime}}}^{2} v_{3} p^{2 a+r-s}+\overline{V^{\prime} v_{2}^{\prime}} v_{3}^{2} p^{2 a}+\overline{v_{2}^{\prime}} v_{4} p^{a+r-s} & \left(\bmod p^{s}\right) \\ -{\overline{v_{2}^{\prime}}}^{2} v_{3} p^{2 a+r-s}+\overline{v_{2}^{\prime}} v_{4} p^{a+r-s}\left(\bmod p^{s}\right) & \text { if } a=\frac{s}{2}\end{cases}
$$

where $V^{\prime}=p^{s-r-a}\left(p^{a} v_{3}+v_{2}^{\prime} v_{4}\right)$.

(vii) $n=n_{s_{\beta} s_{\alpha} s_{\beta}, r, s}$. Then $X\left(n_{s_{\beta} s_{\alpha} s_{\beta}, r, s}\right)$ is nonempty when $s \geq r$. Unfolding the conditions (3.1) and (3.2), we compute

$$
\begin{aligned}
& X\left(n_{s_{\beta} s_{\alpha} s_{\beta}, r, s}\right) \\
& \quad=\left\{\left(0, p^{r}, v_{13} p^{r-s}, v_{14} p^{r-s} ; p^{s}, v_{13}, v_{14}, v_{23},-v_{13},-\left(v_{13}^{2}+v_{14} v_{23}\right) p^{-s}\right)\right\},
\end{aligned}
$$


where $v_{13}, v_{14}, v_{23}\left(\bmod p^{s}\right)$, such that $\left(v_{13}, v_{14}, p^{s}\right)=p^{s-r},\left(v_{14}, p^{s}\right) \mid v_{13}^{2}$, and $\left(p^{s-r}, v_{23}, v_{34}\right)=1$. Recall that $v_{34}=-\left(v_{13}^{2}+v_{14} v_{23}\right) p^{-s}$. We write $v_{13}=$ $v_{13}^{\prime} p^{s-r}, v_{14}=v_{14}^{\prime} p^{s-r}$, so $\left(v_{13}^{\prime}, v_{14}^{\prime}, p^{r}\right)=1$. Bruhat decomposition gives

$$
\begin{aligned}
x & =\left(\begin{array}{cccc}
1 & \beta_{1} & \beta_{2} & \beta_{3} \\
& 1 & \beta_{4} & \beta_{5} \\
& 1 & \\
& -\beta_{1} & 1
\end{array}\right)\left(\begin{array}{c}
p^{r-s} \\
p^{r-r}
\end{array}\right)\left(\begin{array}{ccc}
1 & -v_{23} p^{-s} & v_{13}^{\prime} p^{-r} \\
1 & v_{13}^{\prime} p^{-r} & v_{14}^{\prime} p^{-r} \\
& 1 &
\end{array}\right) \\
& =\left(\begin{array}{cccc}
-\beta_{3} p^{s-r} & \beta_{2} p^{r} & \beta_{2} v_{13}^{\prime}+\beta_{1} p^{r-s}+\beta_{3} v_{23} p^{-r} & \beta_{2} v_{14}^{\prime}-\beta_{3} v_{13}^{\prime} p^{s-2 r}-p^{-r} \\
-\beta_{5} p^{s-r} & \beta_{4} p^{r} & \beta_{4} v_{13}^{\prime}+\beta_{5} v_{23} p^{-r}+p^{r-s} & \beta_{4} v_{14}^{\prime}-\beta_{5} v_{13}^{\prime} p^{s-2 r} \\
0 & p^{r} & v_{13}^{\prime} & v_{14}^{\prime} \\
-p^{s-r} & -\beta_{1} p^{r} & -\beta_{1} v_{13}^{\prime}+v_{23} p^{-r} & -\beta_{1} v_{14}^{\prime}-v_{13}^{\prime} p^{s-2 r}
\end{array}\right) .
\end{aligned}
$$

The entry $-\beta_{1} p^{r}$ being an integer says $\beta_{1}=\beta_{1}^{\prime} p^{-r}$ for $\beta_{1}^{\prime} \in \mathbb{Z}$. Entries $-\beta_{1} v_{13}^{\prime}+$ $v_{23} p^{-r}$ and $-\beta_{1} v_{14}^{\prime}-v_{13}^{\prime} p^{s-2 r}$ being integers says

$$
\beta_{1}^{\prime} v_{13}^{\prime} \equiv v_{23} \quad\left(\bmod p^{r}\right), \quad \beta_{1}^{\prime} v_{14}^{\prime} \equiv-v_{13}^{\prime} p^{s-r} \quad\left(\bmod p^{r}\right) \text {. }
$$

As $\left(v_{13}^{\prime}, v_{14}^{\prime}, p^{r}\right)=1$, this determines $\beta_{1}$ uniquely modulo 1 .

Entries $\beta_{4} p^{r}$ and $-\beta_{5} p^{s-r}$ being integers says $\beta_{4}=\beta_{4}^{\prime} p^{-r}$ and $\beta_{5}=\beta_{5}^{\prime} p^{r-s}$ for some $\beta_{4}^{\prime}, \beta_{5}^{\prime} \in \mathbb{Z}$. The entry $\beta_{4} v_{13}^{\prime}+\beta_{5} v_{23} p^{-r}+p^{r-s}$ being an integer says

$$
\beta_{4}^{\prime} v_{13}^{\prime} p^{s-r}+\beta_{5}^{\prime} v_{23}+p^{r} \equiv 0 \quad\left(\bmod p^{s}\right),
$$

which implies

$$
\beta_{5}^{\prime} v_{23} \equiv-p^{r} \quad\left(\bmod p^{s-r}\right)
$$

The entry $\beta_{4} v_{14}^{\prime}-\beta_{5} v_{13}^{\prime} p^{s-2 r}$ being an integer says

$$
\beta_{4}^{\prime} v_{14}^{\prime} p^{s-r}-\beta_{5}^{\prime} v_{13}^{\prime} p^{s-r} \equiv 0 \quad\left(\bmod p^{s}\right)
$$

Then, $v_{13}^{\prime}$ times (3.12) minus $v_{14}^{\prime}$ times (3.10) gives

$$
\begin{aligned}
\beta_{5}^{\prime}\left(-v_{13}^{\prime}{ }^{2} p^{s-r}-v_{14}^{\prime} v_{23}\right) & \equiv p^{r} v_{14}^{\prime} \quad\left(\bmod p^{s}\right), \\
\beta_{5}^{\prime} p^{r} v_{34} & \equiv p^{r} v_{14}^{\prime} \quad\left(\bmod p^{s}\right), \\
\beta_{5}^{\prime} v_{34} & \equiv v_{14}^{\prime} \quad\left(\bmod p^{s-r}\right) .
\end{aligned}
$$

As $\left(p^{s-r}, v_{23}, v_{34}\right)=1,(3.11)$ and (3.13) determine $\beta_{5}$ uniquely modulo 1 .

So, after writing $u$ for $\beta_{1} p^{r}$ and $\hat{v}_{14}$ for $\beta_{5} p^{s}$, the Kloosterman sum is given by

$$
\mathrm{Kl}_{p}\left(n_{s_{\beta} s_{\alpha} s_{\beta}, r, s}, \psi, \psi^{\prime}\right)=\sum_{\substack{v_{13}, v_{14}, v_{23}\left(\bmod p^{s}\right) \\\left(p^{s}, v_{13}, v_{14}\right)=p^{s-r} \\\left(p^{s}, v_{14}\right) \mid v_{13}^{2} \\\left(p^{s-r}, v_{23}, v_{34}\right)=1}} \mathrm{e}\left(\frac{m_{1} u}{p^{r}}\right) \mathrm{e}\left(\frac{m_{2} \hat{v}_{14}+n_{2} v_{14}}{p^{s}}\right),
$$


where $u$ is chosen modulo $p^{r}$ such that

$$
u v_{13} p^{r-s} \equiv v_{23} \quad\left(\bmod p^{r}\right), \quad u v_{14} p^{r-s} \equiv-v_{13} \quad\left(\bmod p^{r}\right),
$$

and $\hat{v}_{14}$ is chosen modulo $p^{s}$ such that

$$
\hat{v}_{14} v_{23} \equiv-p^{2 r} \quad\left(\bmod p^{s}\right), \quad \hat{v}_{14} v_{34} \equiv v_{14} p^{2 r-s} \quad\left(\bmod p^{s}\right) \text {. }
$$

(viii) $n=n_{w_{0}, r, s}$. Then $X\left(n_{w_{0}, r, s}\right)$ is nonempty whenever $r, s \geq 0$. Unfolding the conditions (3.1) and (3.2), we compute

$X\left(n_{w_{0}, r, s}\right)=\left\{\left(p^{r}, v_{2}, v_{3}, v_{4} ; p^{s}, v_{13}, v_{14},\left(v_{2} v_{13}-v_{3} p^{s}\right) p^{-r},-v_{13},\left(v_{3} v_{14}-v_{4} v_{13}\right) p^{-r}\right)\right\}$

where $v_{2}, v_{3}, v_{4}\left(\bmod p^{r}\right), v_{13}, v_{14}\left(\bmod p^{s}\right)$, such that $v_{13} p^{r}+v_{2} v_{14}-v_{4} p^{s}=$ $0,\left(v_{2}, v_{3}, v_{4}, p^{r}\right)=1$, and $\left(v_{13}, v_{14}, v_{23}, v_{34}, p^{s}\right)=1$. Recall that

$$
v_{23}=\left(v_{2} v_{13}-v_{3} p^{s}\right) p^{-r}, \quad v_{34}=\left(v_{3} v_{14}-v_{4} v_{13}\right) p^{-r} .
$$

Bruhat decomposition gives

$$
\begin{aligned}
x & =\left(\begin{array}{cccc}
1 & \beta_{1} & \beta_{2} & \beta_{3} \\
1 & \beta_{4} & \beta_{5} \\
& & 1 & \\
& -\beta_{1} & 1
\end{array}\right)\left(\begin{array}{ccc} 
& -p^{-r} & \\
& & -p^{r-s} \\
p^{r} & &
\end{array}\right)\left(\begin{array}{cccc}
1 & v_{2} p^{-r} & v_{3} p^{-r} & v_{4} p^{-r} \\
1 & v_{13} p^{-s} & v_{14} p^{-s} \\
& & \\
& & \\
& -v_{2} p^{-r} & 1
\end{array}\right) \\
& =\left(\begin{array}{cccc}
\beta_{2} p^{r} & \beta_{2} v_{2}+\beta_{3} p^{s-r} & \beta_{2} v_{3}+\beta_{3} v_{13} p^{-r}+\beta_{1} v_{2} p^{-s}-p^{-r} & \beta_{2} v_{4}-\beta_{1} p^{r-s}+\beta_{3} v_{14} p^{-r} \\
\beta_{4} p^{r} & \beta_{4} v_{2}+\beta_{5} p^{s-r} & \beta_{4} v_{3}+\beta_{5} v_{13} p^{-r}+v_{2} p^{-s} & \beta_{4} v_{4}+\beta_{5} v_{14} p^{-r}-p^{r-s} \\
p^{r} & v_{2} & v_{3} & v_{4} \\
-\beta_{1} p^{r} & -\beta_{1} v_{2}+p^{s-r} & -\beta_{1} v_{3}+v_{13} p^{-r} & -\beta_{1} v_{4}+v_{14} p^{-r}
\end{array}\right) .
\end{aligned}
$$

The entry $-\beta_{1} p^{r}$ being an integer says $\beta_{1}=\beta_{1}^{\prime} p^{-r}$ for some $\beta_{1}^{\prime} \in \mathbb{Z}$. The last row of $\gamma$ being integral gives

$$
\beta_{1}^{\prime} v_{2} \equiv p^{s} \quad\left(\bmod p^{r}\right), \quad \beta_{1}^{\prime} v_{3} \equiv v_{13} \quad\left(\bmod p^{r}\right), \quad \beta_{1}^{\prime} v_{4} \equiv v_{14} \quad\left(\bmod p^{r}\right) .
$$

As $\left(p^{r}, v_{2}, v_{3}, v_{4}\right)=1$, these equations determine $\beta_{1}$ uniquely modulo 1 .

The entry $\beta_{4} p^{r}$ being an integer says $\beta_{4}=\beta_{4}^{\prime} p^{-r}$ for some $\beta_{4}^{\prime} \in \mathbb{Z}$. Then $\beta_{4} v_{2}+$ $\beta_{5} p^{s-r}$ being an integer says

$$
\beta_{4}^{\prime} v_{2}+\beta_{5} p^{s} \equiv 0 \quad\left(\bmod p^{r}\right)
$$

In particular, this means $\beta_{5}=\beta_{5}^{\prime} p^{-s}$ for some $\beta_{5}^{\prime} \in \mathbb{Z}$. The entries $\beta_{4} v_{3}+$ $\beta_{5} v_{13} p^{-r}+v_{2} p^{-s}$ and $\beta_{4} v_{4}+\beta_{5} v_{14} p^{-r}-p^{r-s}$ being integers says

$$
\beta_{4}^{\prime} v_{3} p^{s}+\beta_{5}^{\prime} v_{13}+v_{2} p^{r} \equiv 0 \quad\left(\bmod p^{r+s}\right)
$$




$$
\beta_{4}^{\prime} v_{4} p^{s}+\beta_{5}^{\prime} v_{14}-p^{2 r} \equiv 0 \quad\left(\bmod p^{r+s}\right)
$$

In particular we deduce

$$
\begin{aligned}
\beta_{5}^{\prime} v_{13}+v_{2} p^{r} \equiv 0 & \left(\bmod p^{s}\right), \\
\beta_{5}^{\prime} v_{14}-p^{2 r} \equiv 0 & \left(\bmod p^{s}\right) .
\end{aligned}
$$

Then, $v_{2}$ times (3.18) minus $v_{3} p^{s}$ times (3.17) gives

$$
\beta_{5}^{\prime}\left(v_{2} v_{13}-v_{3} p^{s}\right)+v_{2}^{2} p^{r} \equiv 0 \quad\left(\bmod p^{r+s}\right),
$$

which implies

$$
\beta_{5}^{\prime} v_{23}+v_{2}^{2} \equiv 0 \quad\left(\bmod p^{s}\right)
$$

Similarly, $v_{3}$ times (3.18) minus $v_{4}$ times (3.19) gives

$$
\beta_{5}^{\prime}\left(v_{3} v_{14}-v_{4} v_{13}\right)-p^{r}\left(v_{3} p^{r}+v_{2} v_{4}\right) \equiv 0 \quad\left(\bmod p^{r+s}\right),
$$

which implies

$$
\beta_{5}^{\prime} v_{34} \equiv\left(v_{3} p^{r}+v_{2} v_{4}\right) \quad\left(\bmod p^{s}\right)
$$

In summary, $\beta_{5}^{\prime}$ satisfies the following equations:

$$
\begin{array}{ll}
\beta_{5}^{\prime} v_{13} \equiv-v_{2} p^{r} \quad\left(\bmod p^{s}\right), & \beta_{5}^{\prime} v_{14} \equiv p^{2 r} \quad\left(\bmod p^{s}\right) \\
\beta_{5}^{\prime} v_{23} \equiv-v_{2}^{2} \quad\left(\bmod p^{s}\right), & \beta_{5}^{\prime} v_{34} \equiv v_{3} p^{r}+v_{2} v_{4} \quad\left(\bmod p^{s}\right)
\end{array}
$$

As $\left(p^{s}, v_{13}, v_{14}, v_{23}, v_{34}\right)=1$, these equations determine $\beta_{5}$ uniquely modulo 1 . So, after writing $\hat{v}_{2}$ for $\beta_{1} p^{r}$ and $\hat{v}_{14}$ for $\beta_{2} p^{s}$, the Kloosterman sum is given by

$$
\mathrm{Kl}_{p}\left(n_{w_{0}, r, s}, \psi, \psi^{\prime}\right)=\sum_{\begin{array}{c}
v_{2}, v_{3}, v_{4}\left(\bmod p^{r}\right) \\
v_{13}, v_{14}\left(\bmod p^{s}\right) \\
v_{13} p^{r}+v_{2} v_{14}-v_{4} p^{s}=0 \\
\left(p^{r}, v_{2}, v_{3}, v_{4}\right)=1 \\
\left(p^{s}, v_{13}, v_{14}, v_{23}, v_{34}\right)=1
\end{array}} \mathrm{e}\left(\frac{m_{1} \hat{v}_{2}+n_{1} v_{2}}{p^{r}}\right) \mathrm{e}\left(\frac{m_{2} \hat{v}_{14}+n_{2} v_{14}}{p^{s}}\right),
$$

where $\hat{v}_{2}$ is chosen modulo $p^{r}$ such that

$$
\hat{v}_{2} v_{2} \equiv p^{s} \quad\left(\bmod p^{r}\right), \quad \hat{v}_{2} v_{3} \equiv v_{13} \quad\left(\bmod p^{r}\right), \quad \hat{v}_{2} v_{4} \equiv v_{14} \quad\left(\bmod p^{r}\right)
$$

and $\hat{v}_{14}$ chosen modulo $p^{s}$ such that

$$
\begin{array}{lll}
\hat{v}_{14} v_{13} \equiv-v_{2} p^{r} \quad\left(\bmod p^{s}\right), & \hat{v}_{14} v_{14} \equiv p^{2 r} \quad\left(\bmod p^{s}\right) \\
\hat{v}_{14} v_{23} \equiv-v_{2}^{2} \quad\left(\bmod p^{s}\right), & \hat{v}_{14} v_{34} \equiv v_{3} p^{r}+v_{2} v_{4} \quad\left(\bmod p^{s}\right)
\end{array}
$$


Now we give a few reduction formulae for Kloosterman sums, which are straightforward to prove.

Proposition 3.2 Let $\psi=\psi_{m_{1}, m_{2}}, \psi^{\prime}=\psi_{n_{1}, n_{2}}$. Then

$$
\begin{aligned}
\mathrm{Kl}_{p}\left(n_{w_{0}, r, 0}, \psi, \psi^{\prime}\right) & =S\left(m_{1}, n_{1} ; p^{r}\right), & \mathrm{Kl}_{p}\left(n_{w_{0}, 0, s}, \psi, \psi^{\prime}\right) & =S\left(m_{2}, n_{2} ; p^{s}\right), \\
\mathrm{Kl}_{p}\left(n_{s_{\alpha} s_{\beta} s_{\alpha}, r, 0}, \psi, \psi^{\prime}\right) & =S\left(m_{1}, 0 ; p^{r}\right), & \mathrm{Kl}_{p}\left(n_{s_{\beta} s_{\alpha} s_{\beta}, 0, s}, \psi, \psi^{\prime}\right) & =S\left(0, m_{2} ; p^{s}\right), \\
\mathrm{Kl}_{p}\left(n_{s_{\alpha} s_{\beta}, r, 0}, \psi, \psi^{\prime}\right) & =S\left(m_{1}, 0 ; p^{r}\right), & \mathrm{Kl}_{p}\left(n_{s_{\beta} s_{\alpha}, 0, s}, \psi, \psi^{\prime}\right) & =S\left(0, m_{2} ; p^{s}\right) .
\end{aligned}
$$

We end the section by proving that the Kloosterman sum attached to the long Weyl element $w_{0}$ is symmetric with respect to characters $\psi, \psi^{\prime}$. Note that this holds for $G=\operatorname{Sp}(2 r)$ in general.

Proposition 3.3 Let $G=\operatorname{Sp}\left(2 r, \mathbb{Q}_{p}\right)$, and $n \in N\left(\mathbb{Q}_{p}\right)$, such that $w(n)=w_{0}$ is the long Weyl element. Let $\psi, \psi^{\prime}: U\left(\mathbb{Q}_{p}\right) / U\left(\mathbb{Z}_{p}\right) \rightarrow \mathbb{C}^{\times}$be characters. Then

$$
\mathrm{K}_{p}\left(n, \psi, \psi^{\prime}\right)=\mathrm{K}_{p}\left(n, \psi^{\prime}, \psi\right)
$$

Proof The definition of Kloosterman sums reads

$$
\mathrm{Kl}_{p}\left(n, \psi, \psi^{\prime}\right)=\sum_{x \in X(n)} \psi(u(x)) \psi^{\prime}\left(u^{\prime}(x)\right)
$$

The key idea of the proof is to find a bijection $X(n) \rightarrow X(n), x \mapsto \tilde{x}$ such that $\psi(u(\tilde{x}))=\psi\left(u^{\prime}(x)\right)$ and $\psi^{\prime}\left(u^{\prime}(\tilde{x})\right)=\psi^{\prime}(u(x))$. Since $w(n)=w_{0}$ is the long Weyl element, $n \in N\left(\mathbb{Q}_{p}\right)$ is of the form

$$
n=\left(D^{-D^{-1}}\right), \quad D=\operatorname{diag}\left(d_{1}, \ldots, d_{r}\right), \quad d_{i} \in \mathbb{Q}_{p}^{\times}
$$

Let $x \in X(n)$, and suppose

$$
u(x)=\left(\begin{array}{cc}
U & S \\
& \left(U^{-1}\right)^{T}
\end{array}\right) \in U\left(\mathbb{Q}_{p}\right), \quad u^{\prime}(x)=\left(\begin{array}{cc}
U^{\prime} & S^{\prime} \\
& \left(U^{\prime-1}\right)^{T}
\end{array}\right) \in U\left(\mathbb{Q}_{p}\right) .
$$

Then we have

$$
x=\left(\begin{array}{cc}
S D U^{\prime} & S D S^{\prime}-U D^{-1}\left(U^{\prime-1}\right)^{T} \\
\left(U^{-1}\right)^{T} D U^{\prime} & \left(U^{-1}\right)^{T} D S^{\prime}
\end{array}\right) \in G\left(\mathbb{Z}_{p}\right) .
$$

Now set

$$
\tilde{u}=\left(\begin{array}{cc}
\left(\tilde{U}^{\prime-1}\right)^{T} & \tilde{S}^{\prime} \\
& \tilde{U}^{\prime}
\end{array}\right), \quad \tilde{u}^{\prime}=\left(\begin{array}{cc}
\left(\tilde{U}^{-1}\right)^{T} & \tilde{S} \\
& \tilde{U}
\end{array}\right)
$$


where

$\tilde{U}_{i j}:=(-1)^{i-j} U_{j i}, \quad \tilde{S}_{i j}:=(-1)^{i-j} S_{j i}, \quad \tilde{U}_{i j}^{\prime}:=(-1)^{i-j} U_{j i}^{\prime}, \quad \tilde{S}_{i j}^{\prime}:=(-1)^{i-j} S_{j i}^{\prime}$.

It is straightforward to verify that $\tilde{u}, \tilde{u}^{\prime} \in U\left(\mathbb{Q}_{p}\right)$. Now set

$$
\tilde{x}=\tilde{u} n \tilde{u}^{\prime}=\left(\begin{array}{cc}
\tilde{S}^{\prime} D\left(\tilde{U}^{-1}\right)^{T} & \tilde{S}^{\prime} D \tilde{S}-\left(\tilde{U}^{\prime-1}\right)^{T} D^{-1} \tilde{U} \\
\tilde{U}^{\prime} D\left(\tilde{U}^{-1}\right)^{T} & \tilde{U}^{\prime} D \tilde{S}
\end{array}\right) \in G\left(\mathbb{Q}_{p}\right)
$$

Now observe

$$
\begin{aligned}
\left(\tilde{S}^{\prime} D\left(\tilde{U}^{-1}\right)^{T}\right)_{i j} & =\sum_{k} \tilde{S}_{i k}^{\prime} d_{k}\left(\tilde{U}^{-1}\right)_{k j}^{T} \\
& =\sum_{k}(-1)^{i+j}\left(U^{-1}\right)_{j k}^{T} d_{k} S_{k i}^{\prime}=(-1)^{i+j}\left(\left(U^{-1}\right)^{T} D S^{\prime}\right)_{j i} \in \mathbb{Z}_{p},
\end{aligned}
$$

and similarly

$$
\begin{gathered}
\left(\tilde{S}^{\prime} D \tilde{S}-\left(\tilde{U}^{\prime-1}\right)^{T} D^{-1} \tilde{U}\right)_{i j}=(-1)^{i+j}\left(S D S^{\prime}-U D^{-1}\left(U^{\prime-1}\right)^{T}\right)_{j i} \in \mathbb{Z}_{p}, \\
\left(\tilde{U}^{\prime} D\left(\tilde{U}^{-1}\right)^{T}\right)_{i j}=(-1)^{i+j}\left(\left(U^{-1}\right)^{T} D U^{\prime}\right)_{j i} \in \mathbb{Z}_{p} .
\end{gathered}
$$

Hence $\tilde{x} \in G\left(\mathbb{Z}_{p}\right)$. Moreover, we may directly verify that $\alpha_{i}(\tilde{u})=\alpha_{i}\left(u^{\prime}\right), \alpha_{i}\left(\tilde{u}^{\prime}\right)=$ $\alpha_{i}(u)$ for $1 \leq i \leq r$. So $\psi(u(\tilde{x}))=\psi\left(u^{\prime}(x)\right)$ and $\psi^{\prime}\left(u^{\prime}(\tilde{x})\right)=\psi^{\prime}(u(x))$. Finally, using the bijection $X(n) \rightarrow X(n), x \mapsto \tilde{x}$, we deduce that

$$
\begin{aligned}
\mathrm{Kl}_{p}\left(n, \psi, \psi^{\prime}\right) & =\sum_{x \in X(n)} \psi(u(x)) \psi^{\prime}\left(u^{\prime}(x)\right) \\
& =\sum_{x \in X(n)} \psi(u(\tilde{x})) \psi^{\prime}\left(u^{\prime}(\tilde{x})\right) \\
& =\sum_{x \in X(n)} \psi^{\prime}(u(x)) \psi\left(u^{\prime}(x)\right)=\mathrm{Kl}_{p}\left(n, \psi^{\prime}, \psi\right)
\end{aligned}
$$

\section{Bounds for Sp(4) Kloosterman sums}

Fix $\psi=\psi_{m_{1}, m_{2}}, \psi^{\prime}=\psi_{n_{1}, n_{2}}$ as in (1.1). We first establish non-trivial bounds for local Kloosterman sums $\mathrm{Kl}_{p}\left(n_{w, r, s}, \psi, \psi^{\prime}\right)$, that is, prove Theorem 1.1.

We start with the local bounds. For $\mathrm{Kl}_{p}\left(n_{\mathrm{id}, 0,0}, \psi, \psi^{\prime}\right)$, there is nothing to prove. Meanwhile, $\mathrm{Kl}_{p}\left(n_{s_{\alpha}, r, 0}, \psi, \psi^{\prime}\right)$ and $\mathrm{Kl}_{p}\left(n_{s_{\beta}, 0, s}, \psi, \psi^{\prime}\right)$ are just GL(2) Kloosterman 
sums. A well-known bound for GL(2) Kloosterman sums is given by [13]

$$
\left|S\left(\mu, v ; p^{k}\right)\right| \leq 2 p^{k / 2}\left(|\mu|_{p}^{-1},|v|_{p}^{-1}, p^{k}\right)^{1 / 2} .
$$

So

$$
\begin{aligned}
& \left|\mathrm{Kl}_{p}\left(n_{s_{\alpha}, r}, \psi, \psi^{\prime}\right)\right|=S\left(m_{1}, n_{1}, p^{r}\right) \ll p^{r / 2}\left(m_{1}, n_{1}, p^{r}\right)^{1 / 2}, \\
& \left|\mathrm{Kl}_{p}\left(n_{s_{\beta}, s}, \psi, \psi^{\prime}\right)\right|=S\left(m_{2}, n_{2}, p^{s}\right) \ll p^{s / 2}\left(m_{2}, n_{2}, p^{s}\right)^{1 / 2}
\end{aligned}
$$

as claimed.

\subsection{Bound for $\mathrm{KI}_{p}\left(n_{s_{\alpha} s_{\beta}, r, s}, \Psi, \psi^{\prime}\right)$}

We recall

$$
\mathrm{Kl}_{p}\left(n_{s_{\alpha} s_{\beta}, r, s}, \psi, \psi^{\prime}\right)=\sum_{\substack{v_{4}\left(\bmod p^{s}\right) \\\left(v_{4}, p^{s}\right)=1}} \sum_{\substack{v_{3}\left(\bmod p^{r}\right) \\\left(v_{3}, p^{r-s}\right)=1}} \mathrm{e}\left(\frac{m_{1} \overline{v_{3}} p^{s}}{p^{r}}\right) \mathrm{e}\left(\frac{m_{2} \overline{v_{4}} v_{3}^{2}+n_{2} v_{4}}{p^{s}}\right)
$$

Without loss of generality, we assume ord ${ }_{p}\left(m_{1}\right) \leq r-s$, and $\operatorname{ord}_{p}\left(m_{2}\right), \operatorname{ord}_{p}\left(n_{2}\right) \leq$ $s$. Observe that

$$
\mathrm{K}_{p}\left(n_{s_{\alpha} s_{\beta}, r, s}, \psi_{m_{1}, m_{2}}, \psi_{n_{1}, n_{2}}\right)=p^{k+2 l} \mathrm{~K}_{p}\left(n_{s_{\alpha} s_{\beta}, r-k-l, s-l} \psi_{m_{1} p^{-k}, m_{2} p^{-l}}, \psi_{n_{1}, n_{2} p^{-l}}\right)
$$

whenever $p^{k} \mid\left(m_{1}, p^{r-s}\right)$ and $p^{l} \mid\left(m_{2}, n_{2}, p^{s}\right)$. So we may assume $s=0, r=s$, or $p \nmid m_{1}\left(m_{2}, n_{2}\right)$.

If $s=0$, then

$$
\left|\mathrm{Kl}\left(n_{s_{\alpha} s_{\beta}, r, 0}, \psi, \psi^{\prime}\right)\right|=\left|\sum_{\substack{v_{3}\left(\bmod p^{r}\right) \\\left(v_{3}, p^{r}\right)=1}} \mathrm{e}\left(\frac{m_{1} \overline{v_{3}}}{p^{r}}\right)\right| \leq p^{\operatorname{ord}_{p}\left(m_{1}\right)} .
$$

If $r=s$, then

$$
\begin{aligned}
\left|\mathrm{Kl}\left(n_{s_{\alpha} s_{\beta}, r, 0}, \psi, \psi^{\prime}\right)\right| & =\left|\sum_{\substack{v_{4}\left(\bmod p^{r}\right) \\
\left(v_{4}, p\right)=1}} \sum_{v_{3}\left(\bmod p^{r}\right)} \mathrm{e}\left(\frac{m_{2} \overline{v_{4}} v_{3}^{2}+n_{2} v_{4}}{p^{r}}\right)\right| \\
& \leq p^{r+\frac{\operatorname{ord} p\left(m_{2}\right)}{2}+\frac{\operatorname{ord} p\left(n_{2}\right)}{2}}
\end{aligned}
$$

is just a summation of quadratic Gauss sums, and is easily evaluated. 
Now suppose $p \nmid m_{1}\left(m_{2}, n_{2}\right)$. If $p \mid m_{2}$ and $s>1$, then

$$
\begin{aligned}
\mathrm{Kl}\left(n_{s_{\alpha} s_{\beta}, r, s}, \psi, \psi^{\prime}\right) & =\sum_{\substack{v_{4}\left(\bmod p^{s-1}\right) \\
\left(v_{4}, p\right)=1}} \sum_{\substack{v_{3}\left(\bmod p^{r}\right) \\
\left(v_{3}, p^{r-s}\right)=1}} \sum_{k=0}^{p-1} \mathrm{e}\left(\frac{m_{1} \overline{v_{3}}}{p^{r-s}}\right) \mathrm{e}\left(\frac{m_{2} \overline{v_{4}} v_{3}^{2}+n_{2}\left(v_{4}+k p^{s-1}\right)}{p^{s}}\right) \\
& =p \sum_{k=0}^{p-1} \mathrm{e}\left(\frac{n_{2} k}{p}\right) \mathrm{Kl}\left(n_{s_{\alpha} s_{\beta}, r-1, s-1}, \psi_{m_{1}, m_{2} / p}, \psi^{\prime}\right)=0 .
\end{aligned}
$$

Now suppose $p \mid m_{2}$ and $s=1$. We may also assume $r \geq 2$. Then

$$
\mathrm{Kl}\left(n_{s_{\alpha} s_{\beta}, r, 1}, \psi, \psi^{\prime}\right)=\sum_{\substack{v_{4}(\bmod p) \\\left(v_{4}, p\right)=1}} \sum_{\substack{v_{3}\left(\bmod p^{r}\right) \\\left(v_{3}, p\right)=1}} \mathrm{e}\left(\frac{m_{1} \overline{v_{3}}}{p^{r-1}}\right) \mathrm{e}\left(\frac{n_{2} v_{4}}{p}\right)= \begin{cases}p & \text { if } r=2 \\ 0 & \text { if } r>2\end{cases}
$$

The same argument shows that the bound also holds when $p \mid n_{2}$. Now assume $p \nmid m_{1} m_{2} n_{2}$. When $p$ is odd, the same argument shows that the sum is zero unless $r=2 s$; when $p=2$, the sum is zero unless $r=2 s$ or $r=2 s-1$.

We first consider the case $r=2 s$. When $s=1$, we have

$$
\mathrm{Kl}_{p}\left(n_{s_{\alpha} s_{\beta}, 2,1}, \psi, \psi^{\prime}\right)=\sum_{\substack{v_{4}(\bmod p) \\\left(v_{4}, p\right)=1}} \sum_{\substack{v_{3}\left(\bmod p^{2}\right) \\\left(v_{3}, p\right)=1}} \mathrm{e}\left(\frac{m_{1} \overline{v_{3}}+m_{2} \overline{v_{4}} v_{3}^{2}+n_{2} v_{4}}{p}\right) .
$$

We apply a theorem of Adolphson and Sperber on exponential sums of Laurent polynomials. Let $k=\mathbb{F}_{q}$ be a finite field of characteristic $p$. Let

$$
f=\sum_{j \in J} a_{j} x^{j} \in k\left[x_{1}, \ldots, x_{n},\left(x_{1} \ldots x_{n}\right)^{-1}\right]
$$

be a Laurent polynomial in $n$ variables. We assume that $a_{j} \neq 0$ for all $j \in J$. Let $\Psi$ be a nontrivial additive character of $k$, we set

$$
S^{*}(f)=\sum_{x \in\left(k^{\times}\right)^{n}} \Psi(f(x))
$$

The Newton polyhedron of $f$, denoted by $\Delta(f)$, is the convex hull in $\mathbb{R}^{n}$ of the set $J \cup\{(0, \ldots, 0)\}$. We denote by $V(f)$ the volume of $\Delta(f)$ with respect to the Lebesgue measure on $\mathbb{R}^{n}$. For a face $\sigma$ (of any dimension) of $\Delta(f)$, we set

$$
f_{\sigma}=\sum_{j \in \sigma \cap J} a_{j} x^{j}
$$


We say that $f$ is non-degenerate with respect to $\Delta(f)$ if for every face $\sigma$ of $\Delta(f)$ that does not contain the origin, the polynomials

$$
\frac{\partial f_{\sigma}}{\partial x_{1}}, \ldots, \frac{\partial f_{\sigma}}{\partial x_{n}}
$$

have no common zeroes in $\left(\bar{k}^{\times}\right)^{n}$, where $\bar{k}$ denotes an algebraic closure of $k$. Then we have the following estimate.

Theorem 4.1 [1, Corollary 4.3] Given an n-dimensional integral polyhedron $\Delta$ in $\mathbb{R}^{n}$, there is a set $\mathscr{S}_{\Delta}$ consisting of all but finitely many prime numbers, such that if $\operatorname{char}(k) \in \mathscr{S}_{\Delta}$, and

$$
f \in k\left[x_{1}, \ldots, x_{n},\left(x_{1} \ldots x_{n}\right)^{-1}\right]
$$

is a non-degenerate Laurent polynomial with $\Delta(f)=\Delta$, then $\left|S^{*}(f)\right| \leq n ! V(f) q^{n / 2}$. Moreover, when $n=2$, the restriction on char $(k)$ can be removed.

Now set $f(x, y)=\frac{m_{1}}{x}+\frac{m_{2} x^{2}}{y}+n_{2} y \in \mathbb{F}_{p}\left[x, y,(x y)^{-1}\right]$, and $\Psi: \mathbb{F}_{p} \rightarrow \mathbb{C}^{\times}$ the standard additive character on $\mathbb{F}_{p}$. Then $p S^{*}(f)=\mathrm{Kl}_{p}\left(n_{s_{\alpha} s_{\beta}, 2,1}, \psi, \psi^{\prime}\right)$. We claim that $f$ is non-degenerate whenever $p \neq 2$. The Newton polyhedron $\Delta(f)$ is the triangle with vertices $(x, y)=(-1,0),(2,-1),(0,1)$, and we evaluate $V(f)=2$. We list the faces $\sigma$ that do not contain the origin, and compute the derivatives $\frac{\partial f_{\sigma}}{\partial x}, \frac{\partial f_{\sigma}}{\partial y}$. We denote by $\left\langle a_{0}, \ldots, a_{j}\right\rangle$ the $j$-dimensional face of $\Delta(f)$ containing $a_{0}, \ldots, a_{j}$. We compute:

$$
\begin{aligned}
\sigma_{1}= & \langle(-1,0),(2,-1)\rangle, f_{\sigma_{1}}=\frac{m_{1}}{x}+\frac{m_{2} x^{2}}{y}, \\
& \left(\frac{\partial f_{\sigma_{1}}}{\partial x}, \frac{\partial f_{\sigma_{1}}}{\partial y}\right)=\left(-\frac{m_{1}}{x^{2}}+\frac{2 m_{2} x}{y},-\frac{m_{2} x^{2}}{y^{2}}\right) ; \\
\sigma_{2}= & ((-1,0),(0,1)\rangle, f_{\sigma_{2}}=\frac{m_{1}}{x}+n_{2} y, \\
& \left(\frac{\partial f_{\sigma_{2}}}{\partial x}, \frac{\partial f_{\sigma_{2}}}{\partial y}\right)=\left(-\frac{m_{1}}{x^{2}}, n_{2}\right) ; \\
\sigma_{3}= & \langle(2,-1),(0,1)\rangle, f_{\sigma_{3}}=\frac{m_{2} x^{2}}{y}+n_{2} y, \\
& \left(\frac{\partial f_{\sigma_{3}}}{\partial x}, \frac{\partial f_{\sigma_{3}}}{\partial y}\right)=\left(\frac{2 m_{2} x}{y},-\frac{m_{2} x^{2}}{y^{2}}+n_{2}\right) ; \\
\sigma_{4}= & \langle(-1,0)\rangle, f_{\sigma_{4}}=\frac{m_{1}}{x}, \\
& \left(\frac{\partial f_{\sigma_{4}}}{\partial x}, \frac{\partial f_{\sigma_{4}}}{\partial y}\right)=\left(-\frac{m_{1}}{x^{2}}, 0\right) ; \\
\sigma_{5}= & \langle(2,-1)\rangle, f_{\sigma_{5}}=\frac{m_{2} x^{2}}{y},
\end{aligned}
$$




$$
\begin{aligned}
& \left(\frac{\partial f_{\sigma_{5}}}{\partial x}, \frac{\partial f_{\sigma_{5}}}{\partial y}\right)=\left(\frac{2 m_{2} x}{y},-\frac{m_{2} x^{2}}{y^{2}}\right) ; \\
\sigma_{6}= & \langle(0,1)\rangle, f_{\sigma_{6}}=n_{2} y, \\
& \left(\frac{\partial f_{\sigma_{6}}}{\partial x}, \frac{\partial f_{\sigma_{6}}}{\partial y}\right)=\left(0, n_{2}\right) .
\end{aligned}
$$

Observe that when $p \neq 2$, the terms $-\frac{m_{1}}{x^{2}}, \frac{2 m_{2} x}{y},-\frac{m_{2} x^{2}}{y^{2}}, n_{2}$ have no zeroes in $\left(\overline{\mathbb{F}}_{p} \times\right)^{2}$. Hence we conclude that $f$ is non-degenerate when $p \neq 2$. Now we apply Theorem 4.1 and conclude that

$$
\left|S^{*}(f)\right| \leq 2 V(f) p=4 p \text {. }
$$

for $p \neq 2$. However, by direct computation, the bound (4.2) also holds for $p=2$. Therefore, for all primes $p$, we have

$$
\left|\mathrm{Kl}_{p}\left(n_{s_{\alpha} s_{\beta}, 2,1}, \psi, \psi^{\prime}\right)\right| \leq 4 p^{2}
$$

So the bound holds in this case.

If $s>1$, we apply the stationary phase method, following [7]. Let $V$ be a smooth scheme of dimension $n$, and $f: V \rightarrow \mathbb{A}^{1}=\mathbb{A}_{\mathbb{Z}_{p}}^{1}$ a $\mathbb{Z}_{p}$-morphism. We consider the exponential sum

$$
S_{m}(f):=\sum_{x \in V\left(\mathbb{Z} / p^{m} \mathbb{Z}\right)} \mathrm{e}\left(\frac{f(x)}{p^{m}}\right) .
$$

Let $j \leq m$ be a positive integer. We write

$$
D\left(\mathbb{Z} / p^{j} \mathbb{Z}\right):=\left\{x \in V\left(\mathbb{Z} / p^{j} \mathbb{Z}\right) \mid \nabla f(x) \equiv 0 \quad\left(\bmod p^{j}\right)\right\}
$$

to denote the "approximate critical points" of $f$. For $\bar{x} \in\left(\mathbb{Z} / p^{j} \mathbb{Z}\right)^{n}$, we define

$$
S_{m}(f)_{\bar{x}}=\sum_{\substack{x \in V\left(\mathbb{Z} / p^{m} \\ \mathbb{Z}\right) \\ x \equiv \bar{x}\left(\bmod p^{j}\right)}} \mathrm{e}\left(\frac{f(x)}{p^{m}}\right) .
$$

Clearly we have

$$
S_{m}(f)=\sum_{\bar{x} \in\left(\mathbb{Z} / p^{j} \mathbb{Z}\right)^{n}} S_{m}(f)_{\bar{x}}
$$

Theorem 4.2 [7, Theorem 1.8(a)] If $2 j \leq m$, then $S_{\bar{x}}=0$ unless $\bar{x} \in D\left(\mathbb{Z} / p^{j} \mathbb{Z}\right)$. Now suppose $m=2 j$ or $2 j+1$, and let $x \in\left(\mathbb{Z} / p^{m} \mathbb{Z}\right)^{n}$ map to $\bar{x} \in D\left(\mathbb{Z} / p^{j} \mathbb{Z}\right)$. If 
$m=2 j$, then we have

$$
S_{m}(f)_{\bar{x}}=p^{m n / 2} \mathrm{e}\left(\frac{f(x)}{p^{m}}\right)
$$

If $m=2 j+1$, then we have

$$
S_{m}(f)_{\bar{x}}=p^{(m-1) n / 2} \mathrm{e}\left(\frac{f(x)}{p^{m}}\right) \sum_{y \in(\mathbb{Z} / p \mathbb{Z})^{n}} \mathrm{e}\left(\frac{\frac{1}{2} y^{T} H_{x} y+p^{-j} \nabla f(x) \cdot y}{p}\right),
$$

where $H_{x}$ is the Hessian matrix of $f$ at $x$. In particular, if we let $t$ denote the maximum value of $n-\operatorname{rank}_{\mathbb{F}_{p}} H_{\bar{x}}$ for $\bar{x} \in D\left(\mathbb{Z} / p^{j} \mathbb{Z}\right)$, then $|S| \leq\left|D\left(\mathbb{Z} / p^{j} \mathbb{Z}\right)\right| p^{(m n+t) / 2}$.

Now we apply the stationary phase method. Let $f(x, y)=\frac{m_{1}}{x}+\frac{m_{2} x^{2}}{y}+n_{2} y$. Consider the sum

$$
S=\sum_{x, y \in\left(\mathbb{Z} / p^{s} \mathbb{Z}\right)^{\times}} \mathrm{e}\left(\frac{f(x, y)}{p^{s}}\right)=p^{-s} \mathrm{Kl}_{p}\left(n_{s_{\alpha} s_{\beta}, 2 s, s}, \psi, \psi^{\prime}\right)
$$

Let $j \geq 1$ be such that $2 j \leq s$. Define as in (4.4)

$$
\begin{aligned}
D\left(\mathbb{Z} / p^{j} \mathbb{Z}\right) & =\left\{(x, y) \in\left(\mathbb{Z} / p^{j} \mathbb{Z}\right)^{\times} \times\left(\mathbb{Z} / p^{j} \mathbb{Z}\right)^{\times} \mid \begin{array}{ll}
\nabla f(x, y) \equiv 0 & \left.\left(\bmod p^{j}\right)\right\}
\end{array}\right. \\
& =\left\{(x, y) \in\left(\mathbb{Z} / p^{j} \mathbb{Z}\right)^{\times} \times\left(\mathbb{Z} / p^{j} \mathbb{Z}\right)^{\times} \mid \begin{array}{ll}
2 m_{2} x^{3} \equiv m_{1} y & \left(\bmod p^{j}\right), \\
m_{2} x^{2} \equiv n_{2} y^{2} & \left(\bmod p^{j}\right)
\end{array}\right\} .
\end{aligned}
$$

It is straightforward to check that $\left|D\left(\mathbb{Z} / p^{j} \mathbb{Z}\right)\right| \leq 4$, and $H_{x, y}$ is invertible over $\mathbb{F}_{p}$ for all $(x, y) \in D\left(\mathbb{Z} / p^{j} \mathbb{Z}\right)$, so $\operatorname{rank}_{\mathbb{F}_{p}} H_{x, y}=2$. So we deduce from Theorem 4.2 that

$$
\left|\mathrm{Kl}_{p}\left(n_{s_{\alpha} s_{\beta}, r, s}, \psi, \psi^{\prime}\right)\right| \leq 4 p^{2 s}
$$

Now it remains to tackle the case $p=2, r=2 s-1$. As $p$ is fixed, it suffices to prove the bound for sufficiently large $s$, so we can always use the stationary phase method. Let $f(x, y)=\frac{2 m_{1}}{x}+\frac{m_{2} x^{2}}{y}+n_{2} y$. Consider the sum

$$
S=\sum_{x, y \in\left(\mathbb{Z} / p^{s} \mathbb{Z}\right)^{\times}} \mathrm{e}\left(\frac{f(x, y)}{p^{s}}\right)=p^{-s+1} \mathrm{Kl}_{p}\left(n_{s_{\alpha} s_{\beta}, 2 s-1, s}, \psi, \psi^{\prime}\right) .
$$

Let $j \geq 1$ be such that $2 j \leq s$. Define as in (4.4) 


$$
\begin{aligned}
& D\left(\mathbb{Z} / p^{j} \mathbb{Z}\right) \\
& \quad=\left\{(x, y) \in\left(\mathbb{Z} / p^{j} \mathbb{Z}\right)^{\times} \times\left(\mathbb{Z} / p^{j} \mathbb{Z}\right)^{\times} \mid \nabla f(x, y) \equiv 0 \quad\left(\bmod p^{j}\right)\right\} \\
& \quad=\left\{(x, y) \in\left(\mathbb{Z} / p^{j} \mathbb{Z}\right)^{\times} \times\left(\mathbb{Z} / p^{j} \mathbb{Z}\right)^{\times} \mid \begin{array}{l}
2 m_{2} x^{3} \equiv 2 m_{1} y \quad\left(\bmod p^{j}\right), \\
m_{2} x^{2} \equiv n_{2} y^{2} \quad\left(\bmod p^{j}\right)
\end{array}\right\} .
\end{aligned}
$$

Then we have $\left|D\left(\mathbb{Z} / p^{j} \mathbb{Z}\right)\right| \leq 16$. The Hessian $H_{x, y}$ is not invertible, but nevertheless we have from Theorem 4.2 that

$$
\left|\mathrm{Kl}_{p}\left(n_{s_{\alpha} s_{\beta}, 2 s-1, s}, \psi, \psi^{\prime}\right)\right| \leq 64 p^{2 s-1}
$$

This finishes the proof of the bound for $\mathrm{Kl}_{p}\left(n_{s_{\alpha} s_{\beta}, r, s}, \psi, \psi^{\prime}\right)$.

\subsection{Bound for $\mathrm{Kl}_{p}\left(n_{s_{\beta} s_{\alpha}, r, s}, \psi, \psi^{\prime}\right)$}

As we have mentioned in Sect. 3, the Kloosterman sum $\mathrm{K}_{p}\left(n_{s_{\beta} s_{\alpha}, r, s}, \psi, \psi^{\prime}\right)$ differs from the GL(3) Kloosterman sum $S\left(n_{1}, m_{1}, m_{2} ; p^{r}, p^{s-r}\right)$ just by a factor of $p^{r}$. So our bound immediately follows from the estimate given by Larsen [2, Appendix], whose proof we omit here.

\subsection{Bound for $\mathrm{KI}_{p}\left(n_{s_{\alpha} s_{\beta} s_{\alpha}, r, s}, \Psi, \psi^{\prime}\right)$}

We make use of the decomposition for Kloosterman sums in Sect. 2 to obtain a nontrivial bound for $\mathrm{Kl}_{p}\left(n_{s_{\alpha} s_{\beta} s_{\alpha}, r, s}, \psi, \psi^{\prime}\right)$.

Let $w=s_{\alpha} s_{\beta} s_{\alpha}$, and $n=n_{s_{\alpha} s_{\beta} s_{\alpha}, r, s}$. Note that we have $s \leq 2 r$. Then $\Delta_{w}=\{\alpha\}$, and

$$
A_{w}(\ell)=\left(\mathbb{Z} / p^{\ell} \mathbb{Z}\right)^{2} \times\left(\mathbb{Z} / p^{\ell} \mathbb{Z}\right)
$$

Let $t=\operatorname{diag}\left(a_{1}, a_{2}, c a_{1}^{-1}, c a_{2}^{-1}\right) \in \mathcal{T}$. Then $s=n^{-1} t n=\operatorname{diag}\left(c a_{1}^{-1}, a_{2}, a_{1}, c a_{2}^{-1}\right)$. We compute

$$
\kappa_{1}^{\prime}(t * x)=c a_{1}^{-1} a_{2}^{-1} \kappa_{1}^{\prime}(x)
$$

So

$$
V_{w}(\ell)=\left\{\left(\lambda, \lambda^{\prime}\right) \in A_{w}(\ell)^{\times} \mid \lambda_{1} \lambda_{2} \lambda_{1}^{\prime}=1\right\}
$$

If $\theta: A_{w}(\ell) \rightarrow \mathbb{C}^{\times}$is given by

$$
\theta\left(\lambda, \lambda^{\prime}\right)=\mathrm{e}\left(\frac{n_{1} \lambda_{1}+n_{2} \lambda_{2}}{p^{\ell}}\right) \text { e }\left(\frac{n_{1}^{\prime} \lambda_{1}^{\prime}}{p^{\ell}}\right), \quad n_{1}, n_{2}, n_{1}^{\prime} \in \mathbb{Z}
$$


then

$$
S_{w}(\theta, \ell)=\sum_{\lambda_{2} \in\left(\mathbb{Z} / p^{\ell} \mathbb{Z}\right)^{\times}} \mathrm{e}\left(\frac{n_{2} \lambda_{2}}{p^{\ell}}\right) S\left(n_{1} \lambda_{2}^{-1}, n_{1}^{\prime} ; p^{\ell}\right) .
$$

Suppose $x_{a, b}^{v_{3}} \in X(n)$ has Plücker coordinates

$$
\left(v_{1}, v_{2}, v_{3}, v_{4} ; v_{14}\right)=\left(p^{r}, p^{r-a}, v_{3}, p^{r-b} ; p^{s}\right) .
$$

Let $\delta=\left(p^{r-a}, p^{a} v_{3}+p^{r-b}\right)$. Then $v_{14}=\frac{p^{r+a}}{\delta}$. This says $s-r \leq a \leq \frac{s}{2}, b \leq r$. Then $\delta=p^{r+a-s}$. Then

$$
u^{\prime}\left(x_{a, b}^{v_{3}}\right)=\left(\begin{array}{ccc}
1 p^{-a} & v_{3} p^{-r} & p^{-b} \\
1 & p^{-b} & \\
& 1 & \\
& -p^{-a} & 1
\end{array}\right) \quad\left(\bmod U\left(\mathbb{Z}_{p}\right)\right)
$$

Let $X_{a, b}^{v_{3}}(n)=\mathcal{T} * x_{a, b}^{v_{3}}$, and define

$$
S_{a, b}^{v_{3}}\left(n, \psi, \psi^{\prime}\right)=\sum_{x \in X_{a, b}^{v_{3}}(n)} \psi(u(x)) \psi^{\prime}\left(u^{\prime}(x)\right) .
$$

We also let

$$
X_{a, b}(n)=\coprod_{\substack{v_{3} \\\left(p^{r-a}, p^{a} v_{3}+p^{r-b}\right)=p^{r+a-s}}} X_{a, b}^{v_{3}(n),}
$$

and

$$
S_{a, b}\left(n, \psi, \psi^{\prime}\right)=\sum_{x \in X_{a, b}(n)} \psi(u(x)) \psi^{\prime}\left(u^{\prime}(x)\right) .
$$

Let $x \in X(n)$, with Plücker coordinates $v_{2}=v_{2, x}, v_{4}=v_{4, x}$. Then $\operatorname{ord}_{p}\left(v_{2, x}\right)=r-a$, $\operatorname{ord}_{p}\left(v_{4, x}\right)=r-b$ for some $s-r \leq a \leq s / 2,0 \leq b \leq r$. So $x$ lies in the $\mathcal{T}$-orbit of $x_{a, b}^{v_{3}}$ for some $v_{3}\left(\bmod p^{r}\right)$, and hence $x \in X_{a, b}(n)$. This gives a partition

$$
X(n)=\coprod_{\substack{s-r \leq a \leq s / 2 \\ 0 \leq b \leq r}} X_{a, b}(n)
$$


As $r \geq \frac{s}{2} \geq a, r \geq b$, we see that $u(x), u^{\prime}(x)$ have entries in $p^{-r} \mathbb{Z}_{p} / \mathbb{Z}_{p}$ for all $x \in X(n)$. Let $\mathcal{S}_{a, b}$ be a finite subset of $\mathbb{Z}_{p}$ such that

$$
X_{a, b}(n)=\coprod_{v_{3} \in \mathcal{S}_{a, b}} X_{a, b}^{v_{3}}(n)
$$

By Theorem 2.4, we have

$$
S_{a, b}\left(n, \psi, \psi^{\prime}\right)=p^{-4 r}\left(1-p^{-1}\right)^{-2} \sum_{v_{3} \in \mathcal{S}_{a, b}}\left|X_{a, b}^{v_{3}}(n)\right| S_{w}\left(\theta_{a, b}^{v_{3}} ; 2 r\right),
$$

where

$$
\theta_{a, b}^{v_{3}}\left(\lambda, \lambda^{\prime}\right)=\mathrm{e}\left(\frac{m_{2} u \lambda_{2}}{p^{s}}\right) \mathrm{e}\left(\frac{m_{1} \hat{v}_{2} \lambda_{1}+n_{1} p^{r-a} \lambda_{1}^{\prime}}{p^{r}}\right)
$$

with $\hat{v}_{2}$ and $u$ given as in (3.7) and (3.8). By (4.5), we have

$$
S_{w}\left(\theta_{a, b}^{v_{3}} ; 2 r\right)=\sum_{x, y \in\left(\mathbb{Z} / p^{2 r} \mathbb{Z}\right)^{\times}} \mathrm{e}\left(\frac{m_{2} u x}{p^{s}}\right) \mathrm{e}\left(\frac{m_{1} \hat{v}_{2} \bar{x} y+n_{1} p^{r-a} \bar{y}}{p^{r}}\right) .
$$

Since the size of the $\mathcal{T}$-orbit of $x_{a, b}^{v_{3}}$ is bounded by $p^{a+b}$, we have

$$
\sum_{v_{3} \in \mathcal{S}_{a, b}}\left|X_{a, b}^{v_{3}}(n)\right| \leq\left|\mathcal{S}_{a, b}\right| p^{a+b} \leq p^{r+a+b}
$$

We estimate the size of $S_{w}\left(\theta_{a, b}^{v_{3}} ; 2 r\right)$ below. We start by computing the order of $\hat{v}_{2}$ and $u$ in (4.6). From (3.7), it is clear that $\operatorname{ord}_{p}\left(\hat{v}_{2}\right)=s-a$. Now we consider $\operatorname{ord}_{p}(u)$. If $a \neq \frac{s}{2}$, then we have (after putting $v_{2}^{\prime}=\overline{v_{2}^{\prime}}=1$ )

$$
\begin{aligned}
u & =p^{a+r-s}\left(-p^{a} v_{3}+v_{4}\right)+\overline{V^{\prime}} v_{3}^{2} p^{2 a} \\
& =p^{a+r-s}\left(p^{a} v_{3}+v_{4}\right)-2 v_{3} p^{2 a+r-s}+\overline{V^{\prime}} v_{3}^{2} p^{2 a} \\
& =p^{2 a+2 r-2 s} V^{\prime}-2 v_{3} p^{2 a+r-s}+\overline{V^{\prime}} v_{3}^{2} p^{2 a} \\
& =p^{2 a} \overline{V^{\prime}}\left(p^{2 r-2 s} V^{\prime 2}-2 p^{r-s} v_{3} V^{\prime}+v_{3}^{2}\right) \\
& =p^{2 a} \overline{V^{\prime}}\left(p^{r-s} V^{\prime}-v_{3}\right)^{2} \\
& =p^{2 a} \overline{V^{\prime}}\left(p^{-a} v_{4}\right)^{2} \\
& =v_{4}^{2} \overline{V^{\prime}}
\end{aligned}
$$


So $\operatorname{ord}_{p}(u)=2(r-b)$. If $a=\frac{s}{2}$, then (again we set $v_{2}^{\prime}=\overline{v_{2}^{\prime}}=1$ )

$$
u=-v_{3} p^{2 a+r-s}+v_{4} p^{a+r-s}=p^{a+r-s}\left(2 v_{4}-\left(p^{a} v_{3}+v_{4}\right)\right) .
$$

This form will be useful in computing $\operatorname{ord}_{p}(u)$, when more conditions are given.

Case I: Suppose $s<r$. We deduce from (3.7) that $\operatorname{ord}_{p}\left(v_{3}\right)=0, \operatorname{ord}_{p}\left(v_{4}\right)=a$, so only terms with $r=a+b$ contribute. When $a \neq \frac{s}{2}$, we have $\operatorname{ord}_{p}(u)=2(r-b)=$ $2 a$. When $a=\frac{s}{2}$, we can still take $\operatorname{ord}_{p}(u)=s=2 a$. So ord $(u)=2 a$ always holds.

(i) Suppose $a \leq \frac{2 s-r}{3}$. Write $u=p^{2 a} u^{\prime}$. Let

$$
t=\min \left\{\operatorname{ord}_{p}\left(m_{2}\right), \operatorname{ord}_{p}\left(m_{1}\right)+2 s-r-3 a, \operatorname{ord}_{p}\left(n_{1}\right)+s-3 a\right\},
$$

and

$$
f(x, y)=p^{-t}\left(m_{2} u^{\prime} y+\frac{m_{1} \hat{v}_{2} p^{s-r-2 a} x}{y}+\frac{n_{1} p^{s-3 a}}{x}\right)=m_{2}^{\prime} y+\frac{m_{1}^{\prime} x}{y}+\frac{n_{1}^{\prime}}{x},
$$

where $m_{1}^{\prime}=m_{1} \hat{v}_{2} p^{s-r-2 a-t}, m_{2}^{\prime}=m_{2} u^{\prime} p^{-t}, n_{1}^{\prime}=n_{1} p^{s-3 a-t}$. Consider the sum

$$
S=\sum_{x, y \in\left(\mathbb{Z} / p^{s-2 a-t} \mathbb{Z}\right)^{\times}} e\left(\frac{f(x, y)}{p^{s-2 a-t}}\right)=p^{2 s-4 a-4 r-2 t} S_{w}\left(\theta_{a, b}^{v_{3}} ; 2 r\right) .
$$

When $s-2 a-t>1$, let $j \geq 1$ be such that $2 j \leq s-2 a-t$. Define as in (4.4)

$$
\begin{aligned}
D\left(\mathbb{Z} / p^{j} \mathbb{Z}\right) & =\left\{(x, y) \in\left(\mathbb{Z} / p^{j} \mathbb{Z}\right)^{\times} \times\left(\mathbb{Z} / p^{j} \mathbb{Z}\right)^{\times} \mid \nabla f(x, y) \equiv 0 \quad\left(\bmod p^{j}\right)\right\} \\
& =\left\{(x, y) \in\left(\mathbb{Z} / p^{j} \mathbb{Z}\right)^{\times} \times\left(\mathbb{Z} / p^{j} \mathbb{Z}\right)^{\times} \mid \begin{array}{lr}
m_{1}^{\prime} x^{2} \equiv n_{1}^{\prime} y & \left(\bmod p^{j}\right), \\
m_{2}^{\prime} y^{2} \equiv m_{1}^{\prime} x & \left(\bmod p^{j}\right)
\end{array}\right\} .
\end{aligned}
$$

Note that at least one of $m_{1}^{\prime}, m_{2}^{\prime}$ and $n_{1}^{\prime}$ is not divisible by $p$. It then follows that $D\left(\mathbb{Z} / p^{j} \mathbb{Z}\right)$ is empty unless ord $p\left(m_{2}\right)=\operatorname{ord}_{p}\left(m_{1}\right)+2 s-r-3 a=\operatorname{ord}_{p}\left(n_{1}\right)+$ $s-3 a=t$. But then

$$
S=p^{4 a+2 t-2 s} \mathrm{~K}_{p}\left(n_{s_{\beta} s_{\alpha}, s-2 a-t, 3 s-6 a-3 t}, \psi_{m_{1}^{\prime}, m_{2}^{\prime}}, \psi_{n_{1}^{\prime}, 0}\right),
$$

with $p \nmid m_{1}^{\prime} m_{2}^{\prime} n_{1}^{\prime}$. So it follows from the bound for $\mathrm{Kl}_{p}\left(n_{s_{\beta} s_{\alpha}, r, s}, \psi, \psi^{\prime}\right)$ that

$$
\left|S_{w}\left(\theta_{a, b}^{v_{3}} ; 2 r\right)\right| \ll p^{4 r+2 a-s+t} .
$$

Now suppose $s-2 a-t=1$. If $p \nmid m_{1}^{\prime} m_{2}^{\prime} n_{1}^{\prime}$, then it follows by the theorem of Deligne [6, Sommes. trig., 7.1.3] that $S \ll p$. When $p$ divides some (but not all) of $m_{1}^{\prime}, m_{2}^{\prime}, n_{1}^{\prime}$, then the sum reduces to a Ramanujan sum, and is easily evaluated that $S \ll p$ as well. So the bound (4.9) also holds for this case. 
Remark. Theorem 4.1, itself a generalisation of Deligne's theorem, also applies to give the same bound.

The bounds for $S_{w}\left(\theta_{a, b}^{v_{3}} ; 2 r\right)$ in other cases are obtained analogously, and we shall omit the repetitive computations thereafter.

(ii) Suppose $a>\frac{2 s-r}{3}$. Write $\hat{v}_{2}=p^{s-a} \hat{v}_{2}^{\prime}$. Let

$$
t=\min \left\{\operatorname{ord}_{p}\left(m_{2}\right)+r+3 a-2 s, \operatorname{ord}_{p}\left(m_{1}\right), \operatorname{ord}_{p}\left(n_{1}\right)+r-s\right\},
$$

and

$$
f(x, y)=p^{-t}\left(m_{2} u p^{r+a-2 s} y+\frac{m_{1} \hat{v}_{2}^{\prime} x}{y}+\frac{n_{1} p^{r-s}}{x}\right)=m_{2}^{\prime} y+\frac{m_{1}^{\prime} x}{y}+\frac{n_{1}^{\prime}}{x},
$$

where $m_{1}^{\prime} \hat{v}_{2}^{\prime} p^{-t}, m_{2}^{\prime}=m_{2} u p^{r+a-2 s-t}, n_{1}^{\prime}=n_{1} p^{r-s-t}$. Then we have

$$
S=\sum_{x, y \in\left(\mathbb{Z} / p^{r+a-s-t} \mathbb{Z}\right)^{\times}} e\left(\frac{f(x, y)}{p^{r+a-s-t}}\right)=p^{2 a-2 r-2 s-2 t} S_{w}\left(\theta_{a, b}^{v_{3}} ; 2 r\right) .
$$

Then we obtain analogously

$$
\left|S_{w}\left(\theta_{a, b}^{v_{3}} ; 2 r\right)\right| \ll p^{3 r-a+s+t} .
$$

Note that we have $\left(p^{r-a}, p^{a}\left(v_{3}+1\right)\right)=p^{r+a-s}$. A necessary condition for this to hold is that $p^{r-s} \mid v_{3}+1$. So $\left|\mathcal{S}_{a, b}\right| \leq p^{s}$. So, from (4.7) we actually have

$$
\sum_{v_{3} \in \mathcal{S}_{a, b}}\left|X_{a, b}^{v_{3}}(n)\right| \leq p^{s+a+b}
$$

Hence

$$
\begin{aligned}
\left|\mathrm{Kl}_{p}\left(n, \psi, \psi^{\prime}\right)\right| & \leq \sum_{\substack{0 \leq a \leq s / 2 \\
b=r-a \\
0}}\left|S_{a, b}\left(n, \psi, \psi^{\prime}\right)\right| \\
& \ll \sum_{\substack{0 \leq a \leq s / 2 \\
b=r-a}} p^{-4 r} p^{s+a+b} S_{w}\left(\theta_{a, b}^{v_{3}} ; 2 r\right) \\
& \ll \sum_{0 \leq a \leq s / 2} \min \left\{p^{r+2 a+\operatorname{ord}_{p}\left(m_{2}\right)}, p^{s-a+\min \left\{s+\operatorname{ord}_{p}\left(m_{1}\right), r+\operatorname{ord}_{p}\left(n_{1}\right)\right\}}\right\} \\
& \ll p^{\frac{r}{3}+\frac{2 s}{3}+\frac{2}{3} \min \left\{\operatorname{ord}_{p}\left(m_{1}\right)+s, \operatorname{ord}_{p}\left(n_{1}\right)+r\right\}+\frac{1}{3} \operatorname{ord}_{p}\left(m_{2}\right)} .
\end{aligned}
$$

Case II: Suppose $s=r$. We deduce from (3.7) that when $a \neq 0$, then $\operatorname{ord}_{p}\left(v_{3}\right)=$ $0, \operatorname{ord}_{p}\left(v_{4}\right) \geq a$. So, only terms with $r \geq a+b$ contribute. When $a \neq \frac{s}{2}$, we have 
$\operatorname{ord}_{p}(u)=2(r-b)$. When $a=\frac{s}{2}$, we still have $\operatorname{ord}_{p}(u) \leq s=2(r-b)$. So $\operatorname{ord}_{p}(u) \leq 2(r-b)$ always holds. We compute

$$
\left|S_{w}\left(\theta_{a, b}^{v_{3}} ; 2 r\right)\right| \ll p^{2 r} \min \left\{p^{3 r-2 b+\operatorname{ord}_{p}\left(m_{2}\right)}, p^{2 r-a+\min \left\{\operatorname{ord}_{p}\left(m_{1}\right), \operatorname{ord}_{p}\left(n_{1}\right)\right\}}\right\} .
$$

Hence

$$
\begin{aligned}
\left|\mathrm{Kl}_{p}\left(n, \psi, \psi^{\prime}\right)\right| & \\
\quad & \sum_{\substack{0 \leq a \leq r / 2 \\
b \leq r-a}}\left|S_{a, b}\left(n, \psi, \psi^{\prime}\right)\right| \\
& \ll \sum_{\substack{0 \leq a \leq s / 2 \\
b \leq r-a}} p^{-4 r} p^{r+a+b}\left(p^{2 r} \min \left\{p^{3 r-2 b+\operatorname{ord}_{p}\left(m_{2}\right)}, p^{2 r-a+\min \left\{\operatorname{ord}_{p}\left(m_{1}\right), \operatorname{ord}_{p}\left(n_{1}\right)\right\}}\right\}\right) \\
& \ll \sum_{\substack{0 \leq a \leq s / 2 \\
b \leq r-a}} p^{-r+a+b} \min \left\{p^{3 r-2 b+\operatorname{ord}_{p}\left(m_{2}\right)}, p^{2 r-a+\min \left\{\operatorname{ord}_{p}\left(m_{1}\right), \operatorname{ord}_{p}\left(n_{1}\right)\right\}}\right\} \\
& \ll p^{\frac{5 r}{3}+\frac{2}{3} \min \left\{\operatorname{ord}_{p}\left(m_{1}\right), \operatorname{ord}_{p}\left(n_{1}\right)\right\}+\frac{1}{3} \operatorname{ord}_{p}\left(m_{2}\right)} .
\end{aligned}
$$

Case III: $2 r>s>r$. We consider the following subcases:

(a) Suppose $a=s-r$. Then the condition $\left(p^{r-a}, p^{a} v_{3}+p^{r-b}\right)=1$ implies $b=r$. So ord $p(u)=0$. We deduce from (3.7) that $\hat{v}_{2}=0$. So

$$
\left|S_{w}\left(\theta_{a, b}^{v_{3}} ; 2 r\right)\right| \ll p^{3 r-s} \min \left\{p^{r+\operatorname{ord}_{p}\left(m_{2}\right)}, p^{2 r+\operatorname{ord}_{p}\left(n_{1}\right)}\right\} .
$$

(b) Suppose $s-r<a<\frac{s}{2}$. Then we deduce from (3.7) that ord ${ }_{p}\left(v_{3}\right)=0$, $\operatorname{ord}_{p}\left(v_{4}\right) \geq$ $a$. So $a+b \leq r$. Meanwhile, as $r+a-s<a$, the condition $\left(p^{r-a}, p^{a} v_{3}+p^{r-b}\right)=$ $p^{r+a-s}$ says $r-b=r+a-s$, which implies $a+b=s>r$, a contradiction. So there is no contribution from this case.

(c) Suppose $a=\frac{s}{2}$. Again, we deduce from (3.7) that $\operatorname{ord}_{p}\left(v_{3}\right)=0, \operatorname{ord}_{p}\left(v_{4}\right) \geq a$. So, only terms with $r \geq a+b$ contribute. In this case, we don't have a good bound for $\operatorname{ord}_{p}(u)$. So

$$
\left|S_{w}\left(\theta_{a, b}^{v_{3}} ; 2 r\right)\right| \ll p^{3 r+\min \left\{\frac{s}{2}+\operatorname{ord}_{p}\left(m_{1}\right), r-\frac{s}{2}+\operatorname{ord}_{p}\left(n_{1}\right)\right\}} .
$$


Hence

$$
\begin{aligned}
\left|\mathrm{Kl}_{p}\left(n, \psi, \psi^{\prime}\right)\right| \leq & \sum_{\substack{s-r \leq a \leq s / 2 \\
b \leq r-a}}\left|S_{a, b}\left(n, \psi, \psi^{\prime}\right)\right| \\
\ll & \sum_{\substack{a=s-r \\
b=r}} p^{-4 r} p^{r+a+b}\left(p^{3 r-s} \min \left\{p^{r+\operatorname{ord}_{p}\left(m_{2}\right)}, p^{2 r+\operatorname{ord}_{p}\left(n_{1}\right)}\right\}\right) \\
& +\sum_{\substack{a=s / 2 \\
b \leq r-s / 2}} p^{-4 r} p^{r+a+b}\left(p^{3 r+\min \left\{\frac{s}{2}+\operatorname{ord}_{p}\left(m_{1}\right), r-\frac{s}{2}+\operatorname{ord}_{p}\left(n_{1}\right)\right\}}\right) \\
& \ll p^{r+\min \left\{\operatorname{ord}_{p}\left(m_{2}\right), r+\operatorname{ord}_{p}\left(n_{1}\right)\right\}}+p^{r+\min \left\{\frac{s}{2}+\operatorname{ord}_{p}\left(m_{1}\right), r-\frac{s}{2}+\operatorname{ord}_{p}\left(n_{1}\right)\right\}} .
\end{aligned}
$$

Case IV: $s=2 r$. In this case, we have $a=r$, and $v_{3}, v_{4}=p^{r-b}$ is arbitrary. We deduce from (3.7) that $\hat{v}_{2}=0$. We consider the following subcases:

(a) Suppose $b=0$. We may assume $v_{4}=0$. Then $\operatorname{ord}_{p}(u)=r+\operatorname{ord}_{p}\left(v_{3}\right)$. We compute

$$
\left|S_{w}\left(\theta_{a, b}^{v_{3}} ; 2 r\right)\right| \ll p^{r} \min \left\{p^{2 r+\operatorname{ord}_{p}\left(v_{3}\right)+\operatorname{ord}_{p}\left(m_{2}\right)}, p^{2 r+\operatorname{ord}_{p}\left(n_{1}\right)}\right\} .
$$

Fix $c \leq r$. Then

$$
\left|\left\{v_{3} \in \mathcal{S}_{a, b} \mid \operatorname{ord}_{p}\left(v_{3}\right)=c\right\}\right| \leq p^{r-c} .
$$

(b) Suppose $b>0$. Then $\operatorname{ord}_{p}(u)=r-b$. We compute

$$
\left|S_{w}\left(\theta_{a, b}^{v_{3}} ; 2 r\right)\right| \ll p^{r} \min \left\{p^{2 r-b+\operatorname{ord}_{p}\left(m_{2}\right)}, p^{2 r+\operatorname{ord}_{p}\left(n_{1}\right)}\right\} .
$$

Hence

$$
\begin{aligned}
&\left|\mathrm{Kl}_{p}\left(n, \psi, \psi^{\prime}\right)\right| \leq \sum_{\substack{a=r / 2 \\
b \leq r}}\left|S_{a, b}\left(n, \psi, \psi^{\prime}\right)\right| \\
& \ll \sum_{\substack{a=r / 2 \\
b=0 \\
c \leq r}} p^{-4 r} p^{r-c+a+b}\left(p^{r} \min \left\{p^{2 r+c+\operatorname{ord}_{p}\left(m_{2}\right)}, p^{2 r+\operatorname{ord}_{p}\left(n_{1}\right)}\right\}\right) \\
&+\sum_{\substack{a=r / 2 \\
b>0}} p^{-4 r} p^{r+a+b}\left(p^{r} \min \left\{p^{2 r-b+\operatorname{ord}_{p}\left(m_{2}\right)}, p^{2 r+\operatorname{ord}_{p}\left(n_{1}\right)}\right\}\right) \\
& \ll p^{r+\min \left\{\operatorname{ord}_{p}\left(m_{2}\right), r+\operatorname{ord}_{p}\left(n_{1}\right)\right\}} .
\end{aligned}
$$

This finishes the proof of the bound for $\mathrm{K}_{p}\left(n_{s_{\alpha} s_{\beta} s_{\alpha}, r, s}, \psi, \psi^{\prime}\right)$. 


\subsection{Bound for $\mathrm{KI}_{p}\left(n_{s_{\beta} s_{\alpha} s_{\beta}, r, s}, \psi, \psi^{\prime}\right)$}

We make use of the decomposition for Kloosterman sums in Sect. 2 to obtain a nontrivial bound for $\mathrm{Kl}_{p}\left(n_{s_{\beta} s_{\alpha} s_{\beta}, r, s}, \psi, \psi^{\prime}\right)$. and

Let $w=s_{\beta} s_{\alpha} s_{\beta}$, and $n=n_{s_{\beta} s_{\alpha} s_{\beta}, r, s}$. Note that we have $r \leq s$. Then $\Delta_{w}=\{\beta\}$,

$$
A_{w}(\ell)=\left(\mathbb{Z} / p^{\ell} \mathbb{Z}\right)^{2} \times\left(\mathbb{Z} / p^{\ell} \mathbb{Z}\right)
$$

Let $t=\operatorname{diag}\left(a_{1}, a_{2}, c a_{1}^{-1}, c a_{2}^{-1}\right) \in \mathcal{T}$. Then $s=n^{-1} t n=\operatorname{diag}\left(c a_{2}^{-1}, c a_{1}^{-1}, a_{2}, a_{1}\right)$. We compute

$$
\kappa_{2}^{\prime}(t * x)=c a_{1}^{-2} \kappa_{2}^{\prime}(x)
$$

So

$$
V_{w}(\ell)=\left\{\left(\lambda, \lambda^{\prime}\right) \in A_{w}(\ell)^{\times} \mid \lambda_{1}^{2} \lambda_{2} \lambda_{2}^{\prime}=1\right\}
$$

If $\theta: A_{w}(\ell) \rightarrow \mathbb{C}^{\times}$is given by

$$
\theta\left(\lambda, \lambda^{\prime}\right)=\mathrm{e}\left(\frac{n_{1} \lambda_{1}+n_{2} \lambda_{2}}{p^{\ell}}\right) \mathrm{e}\left(\frac{n_{2}^{\prime} \lambda_{2}^{\prime}}{p^{\ell}}\right), \quad n_{1}, n_{2}, n_{2}^{\prime} \in \mathbb{Z},
$$

then

$$
S_{w}(\theta, \ell)=\sum_{\lambda_{1} \in\left(\mathbb{Z} / p^{\ell} \mathbb{Z}\right)^{\times}} \mathrm{e}\left(\frac{n_{1} \lambda_{1}}{p^{\ell}}\right) S\left(n_{2} \lambda_{1}^{-2}, n_{2}^{\prime} ; p^{\ell}\right) .
$$

Suppose $x_{a, b}^{v_{23}} \in X(n)$ has Plücker coordinates

$$
\left(v_{12}, v_{13}, v_{14}, v_{23}\right)=\left(p^{s}, p^{s-a}, p^{s-b}, v_{23}\right)
$$

The condition $\left(v_{12}, v_{14}\right) \mid v_{13}^{2}$ says $s-b \leq 2(s-a)$, that is, $2 a-b \leq s$. We also have $\max \{a, b\}=r$. Then

$$
u^{\prime}\left(x_{a, b}^{v_{23}}\right)=\left(\begin{array}{ccc}
1 & -v_{23} p^{-s} & p^{-a} \\
1 & p^{-a} & p^{-b} \\
& 1 & \\
& & 1
\end{array}\right) \quad\left(\bmod U\left(\mathbb{Z}_{p}\right)\right) .
$$

Let $X_{a, b}^{v_{23}}(n)=\mathcal{T} * x_{a, b}^{v_{23}}$, and define

$$
S_{a, b}^{v_{23}}\left(n, \psi, \psi^{\prime}\right)=\sum_{x \in X_{a, b}^{v_{23}}(n)} \psi(u(x)) \psi^{\prime}\left(u^{\prime}(x)\right)
$$


We also let

$$
X_{a, b}(n)=\coprod_{\left(p^{s-r}, v_{23}, p^{-b} v_{23}-p^{s-2 a}\right)=1} X_{a, b}^{v_{23}(n),}
$$

and

$$
S_{a, b}\left(n, \psi, \psi^{\prime}\right)=\sum_{x \in X_{a, b}(n)} \psi(u(x)) \psi^{\prime}\left(u^{\prime}(x)\right)
$$

Again we have a partition

$$
X(n)=\coprod_{\substack{0 \leq a, b \leq r \\ \max \{a, b\}=r \\ 2 a-b \leq s}} X_{a, b}(n) .
$$

It is clear that $u(x), u^{\prime}(x)$ have entries in $p^{-s} \mathbb{Z}_{p} / \mathbb{Z}_{p}$ for all $x \in X(n)$. Let $\mathcal{S}_{a, b}$ be a finite subset of $\mathbb{Z}_{p}$ such that

$$
X_{a, b}(n)=\coprod_{v_{23} \in \mathcal{S}_{a, b}} X_{a, b}^{v_{23}}(n)
$$

By Theorem 2.4, we have

$$
S_{a, b}\left(n, \psi, \psi^{\prime}\right)=p^{-2 s}\left(1-p^{-1}\right)^{-2} \sum_{v_{23} \in \mathcal{S}_{a, b}}\left|X_{a, b}^{v_{23}}(n)\right| S_{w}\left(\theta_{a, b}^{v_{23}} ; s\right),
$$

where

$$
\theta_{a, b}^{v_{23}}\left(\lambda, \lambda^{\prime}\right)=\mathrm{e}\left(\frac{m_{1} u \lambda_{1}}{p^{r}}\right) \text { e }\left(\frac{m_{2} \hat{v}_{14} \lambda_{2}+n_{2} p^{s-b} \lambda_{2}^{\prime}}{p^{s}}\right)
$$

with $\hat{v}_{14}$ and $u$ given as in (3.14) and (3.15). By (4.10), we have

$$
S_{w}\left(\theta_{a, b}^{v_{23}} ; s\right)=\sum_{x, y \in\left(\mathbb{Z} / p^{s} \mathbb{Z}\right)^{\times}} \mathrm{e}\left(\frac{m_{1} u \bar{x}}{p^{r}}\right) \mathrm{e}\left(\frac{m_{2} \hat{v}_{14} x^{2} \bar{y}+n_{2} p^{s-b} y}{p^{s}}\right) .
$$

Since the size of the $\mathcal{T}$-orbit of $x_{a, b}^{v_{23}}$ is bounded by $p^{a+b}$, we have

$$
\sum_{v_{23} \in \mathcal{S}_{a, b}}\left|X_{a, b}^{v_{23}}(n)\right| \leq\left|\mathcal{S}_{a, b}\right| p^{a+b} \leq p^{s+a}
$$


We estimate the size of $S_{w}\left(\theta_{a, b}^{v_{23}} ; s\right)$. We start by computing the order of $\hat{v}_{14}$ and $u$ in (4.11). From (3.14), we see that

$$
u p^{r-a} \equiv v_{23} \quad\left(\bmod p^{r}\right), \quad u p^{r-b} \equiv-p^{s-a} \quad\left(\bmod p^{r}\right) .
$$

So, if $a=r$, then $u \equiv v_{23}\left(\bmod p^{r}\right)$, and if $b=r$, then $u \equiv-p^{s-a}\left(\bmod p^{r}\right)$. (Recall that $\max \{a, b\}=r$.) Also, we know that

$$
v_{23}=-p^{s-2 a+b}+\beta p^{b}
$$

for some $\beta \in \mathbb{Z}$ such that $\left(\beta, p^{s-2 r+b}\right)=1$ (see [12, Sect. 3.2]). Meanwhile, from (3.15), we see that unless $r=s$, we have $\operatorname{ord}_{p}\left(\hat{v}_{14}\right)=2 r-b$.

Case I: Suppose $r<\frac{s}{2}$. We deduce from (4.14) that $\operatorname{ord}_{p}\left(v_{23}\right)=b$. From (4.13), we deduce $a \geq b$. So we actually have $a=r$, and then $\operatorname{ord}_{p}(u)=b$.

(i) Suppose $b \leq \frac{3 r-s}{2}$. Write $u=p^{b} u^{\prime}$. Let

$$
t=\min \left\{\operatorname{ord}_{p}\left(m_{1}\right), \operatorname{ord}_{p}\left(m_{2}\right)+3 r-2 b-s, \operatorname{ord}_{p}\left(n_{2}\right)+r-2 b\right\}
$$

and

$$
f(x, y)=p^{-t}\left(\frac{m_{1} u^{\prime}}{x}+\frac{m_{2} \hat{v}_{14} p^{r-b-s} x^{2}}{y}+n_{2} p^{r-2 b} y\right)=\frac{m_{1}^{\prime}}{x}+\frac{m_{2}^{\prime} x^{2}}{y}+n_{2}^{\prime} y,
$$

where $m_{1}^{\prime}=m_{1} u^{\prime} p^{-t}, m_{2}^{\prime}=m_{2} \hat{v}_{14} p^{r-b-s-t}, n_{2}^{\prime}=n_{2} p^{r-2 b-t}$. Consider the sum

$$
S=\sum_{x, y \in\left(\mathbb{Z} / p^{r-b-t} \mathbb{Z}\right)^{\times}} e\left(\frac{f(x, y)}{p^{r-b-t}}\right)=p^{2 r-2 s-2 b-2 t} S_{w}\left(\theta_{a, b}^{v_{23}} ; s\right) .
$$

When $r-b-t>1$, let $j \geq 1$ be such that $2 j \leq r-b-t$. Define as in (4.4)

$$
\begin{aligned}
D\left(\mathbb{Z} / p^{j} \mathbb{Z}\right) & =\left\{(x, y) \in\left(\mathbb{Z} / p^{j} \mathbb{Z}\right)^{\times} \times\left(\mathbb{Z} / p^{j} \mathbb{Z}\right)^{\times} \mid \nabla f(x, y) \equiv 0 \quad\left(\bmod p^{j}\right)\right\} \\
& =\left\{(x, y) \in\left(\mathbb{Z} / p^{j} \mathbb{Z}\right)^{\times} \times\left(\mathbb{Z} / p^{j} \mathbb{Z}\right)^{\times} \mid \begin{array}{cc}
2 m_{2}^{\prime} x^{3} \equiv m_{1}^{\prime} y & \left(\bmod p^{j}\right) \\
m_{2}^{\prime} x^{2} \equiv n_{2}^{\prime} y^{2} & \left(\bmod p^{j}\right)
\end{array}\right\} .
\end{aligned}
$$

Note that at least one of $m_{1}^{\prime}, m_{2}^{\prime}$ and $n_{2}^{\prime}$ is not divisible by $p$. It then follows that when $p$ is odd, $D\left(\mathbb{Z} / p^{j} \mathbb{Z}\right)$ is empty unless ord ${ }_{p}\left(m_{1}\right)=\operatorname{ord}_{p}\left(m_{2}\right)+3 r-2 b-s=$ $\operatorname{ord}_{p}\left(n_{2}\right)+r-2 b=t$. But then

$$
S=p^{t+b-r} \mathrm{~K}_{p}\left(n_{s_{\alpha} s_{\beta}, 2 r-2 b-2 t, r-b-t}, \psi_{m_{1}^{\prime}, m_{2}^{\prime}}, \psi_{0, n_{2}^{\prime}}\right),
$$

with $p \nmid m_{1}^{\prime} m_{2}^{\prime} n_{2}^{\prime}$. So it follows from the bound for $\mathrm{Kl}_{p}\left(n_{s_{\alpha} s_{\beta}, r, s}, \psi, \psi^{\prime}\right)$ that

$$
\left|S_{w}\left(\theta_{a, b}^{v_{23}} ; s\right)\right| \ll p^{2 s-r+b+t} .
$$


When $p=2, D\left(\mathbb{Z} / p^{j} \mathbb{Z}\right)$ is empty unless ord $p\left(m_{1}\right)-1=\operatorname{ord}_{p}\left(m_{2}\right)+3 r-2 b-s=$ $\operatorname{ord}_{p}\left(n_{2}\right)+r-2 b=t$. Then

$$
S=p^{t+b-r+1} \mathrm{Kl}_{p}\left(n_{s_{\alpha} s_{\beta}, 2 r-2 b-2 t-1, r-b-t}, \psi_{m_{1}^{\prime} / 2, m_{2}^{\prime}}, \psi_{0, n_{2}^{\prime}}\right),
$$

with $p \nmid\left(m_{1}^{\prime} / 2\right) m_{2}^{\prime} n_{2}^{\prime}$. Again, from the bound for $\mathrm{Kl}_{p}\left(n_{s_{\alpha} s_{\beta}, r, s}, \psi, \psi^{\prime}\right)$, we see that (4.15) also holds for this case.

Now suppose $r-b-t=1$. If $p \nmid m_{1}^{\prime} m_{2}^{\prime} n_{1}^{\prime}$, then it again follows from Theorem 4.1 that $|S| \ll p$. When $p$ divides some (but not all) of $m_{1}^{\prime}, m_{2}^{\prime}, n_{1}^{\prime}$, then the sum reduces to Gauß sums or Ramanujan sums, and is easily evaluated that $|S| \ll p$ as well. So the bound (4.15) also holds for this case.

The bounds for $S_{w}\left(\theta_{a, b}^{v_{23}} ; s\right)$ in other cases are obtained analogously, and we shall omit the repetitive computations thereafter.

(ii) Suppose $b>\frac{3 r-s}{2}$. Write $\hat{v}_{14}=p^{2 r-b} \hat{v}_{14}^{\prime}$. Let

$$
t=\min \left\{\operatorname{ord}_{p}\left(m_{1}\right)+s+2 b-3 r, \operatorname{ord}_{p}\left(m_{2}\right), \operatorname{ord}_{p}\left(n_{2}\right)+s-2 r\right\},
$$

and

$$
f(x, y)=p^{-t}\left(\frac{m_{1} u p^{s+b-3 r}}{x}+\frac{m_{2} \hat{v}_{14}^{\prime} x^{2}}{y}+n_{2} p^{s-2 r} y\right)=\frac{m_{1}^{\prime}}{x}+\frac{m_{2}^{\prime} x^{2}}{y}+n_{2}^{\prime} y,
$$

where $m_{1}^{\prime}=m_{1} u p^{s+b-3 r-t}, m_{2}^{\prime}=m_{2} \hat{v}_{14}^{\prime} p^{-t}, n_{2}^{\prime}=n_{2} p^{s-2 r-t}$. Then we have

$$
S=\sum_{x, y \in\left(\mathbb{Z} / p^{s+b-2 r-t} \mathbb{Z}\right)^{\times}} e\left(\frac{f(x, y)}{p^{s+b-2 r-t}}\right)=p^{2 b-4 r-2 t} S_{w}\left(\theta_{a, b}^{v_{23}} ; s\right) .
$$

Then we obtain analogously

$$
\left|S_{w}\left(\theta_{a, b}^{v_{23}} ; s\right)\right| \ll p^{s+2 r-b+t} .
$$

Hence

$$
\begin{aligned}
& \left|\mathrm{Kl}_{p}\left(n, \psi, \psi^{\prime}\right)\right| \\
& \quad \leq \sum_{\substack{a=r \\
0 \leq b \leq r}}\left|S_{a, b}\left(n, \psi, \psi^{\prime}\right)\right| \\
& \quad \ll \sum_{\substack{a=r \\
0 \leq b \leq r}} p^{-2 s} p^{s+a} \mid S_{w}\left(\theta_{a, b}^{\left.v_{23} ; s\right) \mid}\right. \\
& \quad \ll \sum_{\substack{a=r \\
0 \leq b \leq r}} p^{-2 s} p^{s+a}\left(p^{s-r} \min \left\{p^{s+b+\operatorname{ord}_{p}\left(m_{1}\right)}, p^{r-b+\min \left\{2 r+\operatorname{ord}_{p}\left(m_{2}\right), s+\operatorname{ord}_{p}\left(n_{2}\right)\right\}}\right\}\right) \\
& \quad \ll p^{\frac{s}{2}+\frac{r}{2}+\frac{1}{2} \min \left\{2 r+\operatorname{ord}_{p}\left(m_{2}\right), s+\operatorname{ord}_{p}\left(n_{2}\right)\right\}+\frac{1}{2} \operatorname{ord}_{p}\left(m_{1}\right)} .
\end{aligned}
$$


Case II: Suppose $r=\frac{s}{2}$. We consider the following subcases:

(a) Suppose $b=r$. From (4.13), we may assume $u=0$. We compute

$$
\left|S_{w}\left(\theta_{a, b}^{v_{22}} ; s\right)\right| \ll p^{\frac{3 s}{2}+\min \left\{\operatorname{ord}_{p}\left(m_{2}\right), \operatorname{ord}_{p}\left(n_{2}\right)\right\}} .
$$

(b) Suppose $b<r$. Then $a=r$. From (4.14), we see that $v_{23}=(\beta-1) p^{b}$ for some $\beta \in \mathbb{Z}$ such that $\left(\beta, p^{b}\right)=1$. So ord ${ }_{p}\left(v_{23}\right) \geq b$. And from (4.13), we deduce that $\operatorname{ord}_{p}(u)=\operatorname{ord}_{p}\left(v_{23}\right)$. We compute

$$
\left|S_{w}\left(\theta_{a, b}^{v_{23}} ; s\right)\right| \ll p^{s / 2} \min \left\{p^{s+\operatorname{ord}_{p}\left(v_{23}\right)+\operatorname{ord}_{p}\left(m_{1}\right)}, p^{\frac{3 s}{2}-b+\min \left\{\operatorname{ord}_{p}\left(m_{2}\right), \operatorname{ord}_{p}\left(n_{2}\right)\right\}}\right\} .
$$

Fix $c \geq b$. Then

$$
\left|\left\{v_{23} \in \mathcal{S}_{a, b} \mid \operatorname{ord}_{p}\left(v_{23}\right)=c\right\}\right| \leq p^{s-c} .
$$

Hence

$$
\begin{aligned}
& \left|\mathrm{Kl}_{p}\left(n, \psi, \psi^{\prime}\right)\right| \leq \sum_{\substack{a, b \leq r \\
\max \{a, b\}=r}}\left|S_{a, b}\left(n, \psi, \psi^{\prime}\right)\right| \\
& \quad \ll \sum_{\substack{b=r \\
a \leq r}} p^{-2 s} p^{s+a}\left(p^{\frac{3 s}{2}+\min \left\{\operatorname{ord}_{p}\left(m_{2}\right), \operatorname{ord}_{p}\left(n_{2}\right)\right\}}\right) \\
& \quad+\sum_{\substack{a=r \\
b<r \\
b \leq c \leq r}} p^{-2 s} p^{s-c+a+b}\left(p^{s / 2} \min \left\{p^{s+\operatorname{ord}_{p}\left(v_{23}\right)+\operatorname{ord}_{p}\left(m_{1}\right)}, p^{\frac{3 s}{2}-b+\min \left\{\operatorname{ord}_{p}\left(m_{2}\right), \operatorname{ord}_{p}\left(n_{2}\right)\right\}}\right\}\right) \\
& \ll p^{\frac{5 s}{4}+\frac{1}{2} \operatorname{ord}_{p}\left(m_{1}\right)+\frac{1}{2} \min \left\{\operatorname{ord}_{p}\left(m_{2}\right), \operatorname{ord}_{p}\left(n_{2}\right)\right\} .}
\end{aligned}
$$

Case III: Suppose $s>r>\frac{s}{2}$. We consider the following subcases:

(a) Suppose $b=r$. Then ord $p(u)=s-a$, and $\operatorname{ord}_{p}\left(\hat{v}_{14}\right)=r$. We compute

$$
\left|S_{w}\left(\theta_{a, b}^{v_{23}} ; s\right)\right| \ll p^{s-r} \min \left\{p^{2 s-a+\operatorname{ord}_{p}\left(m_{1}\right)}, p^{r+\min \left\{r+\operatorname{ord}_{p}\left(m_{2}\right)\right\}, s-r+\operatorname{ord}_{p}\left(n_{2}\right)}\right\} .
$$

(b) Suppose $b<r$. Then $a=r$. Then from (4.14) we deduce that $\operatorname{ord}_{p}\left(v_{23}\right)=$ $p^{s-2 r+b}$, and hence ord $p(u)=p^{s-2 r+b}$. We compute

$$
\left|S_{w}\left(\theta_{a, b}^{v_{23}} ; s\right)\right| \ll p^{s-r} \min \left\{p^{2 s-2 r+b+\operatorname{ord}_{p}\left(m_{1}\right)}, p^{r-b+\min \left\{2 r+\operatorname{ord}_{p}\left(m_{2}\right), s+\operatorname{ord}_{p}\left(n_{2}\right)\right\}}\right\} .
$$


Hence

$$
\begin{aligned}
& \left|\mathrm{Kl}_{p}\left(n, \psi, \psi^{\prime}\right)\right| \leq \sum_{\substack{a, b \leq r \\
\max \{a, b\}=r \\
2 a-b \leq s}}\left|S_{a, b}\left(n, \psi, \psi^{\prime}\right)\right| \\
& \quad \ll \sum_{\substack{b=r \\
a \leq r}} p^{-2 s} p^{s+a}\left(p^{s-r} \min \left\{p^{2 s-a+\operatorname{ord}_{p}\left(m_{1}\right)}, p^{r+\min \left\{r+\operatorname{ord}_{p}\left(m_{2}\right)\right\}, s-r+\operatorname{ord}_{p}\left(n_{2}\right)}\right\}\right) \\
& +\sum_{\substack{a=r \\
2 r-s \leq b<r}} p^{-2 s} p^{s+a}\left(p^{s-r} \min \left\{p^{2 s-2 r+b+\operatorname{ord}_{p}\left(m_{1}\right)}, p^{r-b+\min \left\{2 r+\operatorname{ord}_{p}\left(m_{2}\right), s+\operatorname{ord}_{p}\left(n_{2}\right)\right\}}\right\}\right) \\
& \ll p^{s-\frac{r}{2}+\frac{1}{2} \operatorname{ord}_{p}\left(m_{1}\right)+\frac{1}{2} \min \left\{2 r+\operatorname{ord}_{p}\left(m_{2}\right), s+\operatorname{ord}_{p}\left(n_{2}\right)\right\} .}
\end{aligned}
$$

Case IV: $r=s$. In this case we only have to consider terms with $b=r$. Indeed, if $b<r$, then $a=r$, and then by (4.13), we see that $u p^{r-b} \equiv-1\left(\bmod p^{r}\right)$, which says $b=r$, a contradiction. When $b=r$, we have ord $p(u)=s-a$, and from (3.15) we may assume $\hat{v}_{14}=0$. We compute

$$
\left|S_{w}\left(\theta_{a, b}^{v_{23}} ; s\right)\right| \ll \min \left\{p^{2 s-a+\operatorname{ord}_{p}\left(m_{1}\right)}, p^{s+\operatorname{ord}_{p}\left(n_{2}\right)}\right\} .
$$

Hence

$$
\begin{aligned}
\left|\mathrm{Kl}_{p}\left(n, \psi, \psi^{\prime}\right)\right| & \leq \sum_{\substack{b=s \\
a \leq s}}\left|S_{a, b}\left(n, \psi, \psi^{\prime}\right)\right| \\
& \ll \sum_{\substack{b=s \\
a \leq s}} p^{-2 s} p^{s+a}\left(\min \left\{p^{2 s-a+\operatorname{ord}_{p}\left(m_{1}\right)}, p^{s+\operatorname{ord}_{p}\left(n_{2}\right)}\right\}\right) \\
& \ll p^{s+\min \left\{\operatorname{ord}_{p}\left(m_{1}\right), \operatorname{ord}_{p}\left(n_{2}\right)\right\}} .
\end{aligned}
$$

This finishes the proof of the bound for $\mathrm{Kl}_{p}\left(n_{s_{\beta} s_{\alpha} s_{\beta}, r, s}, \psi, \psi^{\prime}\right)$.

\subsection{Bounds for $\mathrm{KI}_{p}\left(n_{w_{0}, r, s}, \psi, \psi^{\prime}\right)$}

We show that under the stratification introduced in Sect. $2, \mathrm{Kl}_{p}\left(n_{w_{0}, r, s}, \psi, \psi^{\prime}\right)$ decomposes into a sum of products of GL(2) Kloosterman sums. So the Kloosterman sum can be bounded using (4.1).

Let $w=w_{0}$, and $n=n_{w_{0}, s, r}$. Then $\Delta_{w_{0}}=\Delta$, and

$$
A_{w_{0}}(\ell)=\left(\mathbb{Z} / p^{\ell} \mathbb{Z}\right)^{2} \times\left(\mathbb{Z} / p^{\ell} \mathbb{Z}\right)^{2}
$$


Let $t=\operatorname{diag}\left(a_{1}, a_{2}, c a_{1}^{-1}, c a_{2}^{-1}\right) \in \mathcal{T}$. Then $s=n^{-1} t n=\operatorname{diag}\left(c a_{1}^{-1}, c a_{2}^{-1}, a_{1}, a_{2}\right)$.

We compute

$$
\kappa_{1}^{\prime}(t * x)=a_{2} a_{1}^{-1} \kappa_{1}^{\prime}(x), \quad \kappa_{2}^{\prime}(t * x)=c a_{2}^{-2} \kappa_{2}^{\prime}(x) .
$$

So

$$
V_{w_{0}}(\ell)=\left\{\left(\lambda, \lambda^{\prime}\right) \in A_{w_{0}}(\ell)^{\times} \mid \lambda_{1} \lambda_{1}^{\prime}=1, \lambda_{2} \lambda_{2}^{\prime}=1\right\}
$$

If $\theta: A_{w_{0}}(\ell) \rightarrow \mathbb{C}^{\times}$is given by

$$
\theta\left(\lambda, \lambda^{\prime}\right)=\prod_{i=1}^{2} \mathrm{e}\left(\frac{n_{i} \lambda_{i}}{p^{\ell}}\right) \prod_{i=1}^{2} \mathrm{e}\left(\frac{n_{i}^{\prime} \lambda_{i}^{\prime}}{p^{\ell}}\right), \quad n_{1}, n_{2}, n_{1}^{\prime}, n_{2}^{\prime} \in \mathbb{Z},
$$

then

$$
S_{w_{0}}(\theta ; \ell)=S\left(n_{1}, n_{1}^{\prime} ; p^{\ell}\right) S\left(n_{2}, n_{2}^{\prime} ; p^{\ell}\right)
$$

Suppose $x_{a, b}^{v_{3}, v_{4}, v_{13}} \in X(n)$ has Plücker coordinates

$$
\left(v_{1}, v_{2}, v_{3}, v_{4} ; v_{12}, v_{13}, v_{14}\right)=\left(p^{r}, p^{r-a}, v_{3}, v_{4} ; p^{s}, v_{13}, p^{s-b}\right)
$$

Note that this also says $r \geq a, s \geq b$. Then

$$
u^{\prime}\left(x_{a, b}^{v_{3}, v_{4}, v_{13}}\right)=\left(\begin{array}{ccc}
1 p^{-a} & v_{3} p^{-r} & v_{4} p^{-r} \\
1 & v_{13} p^{-s} & p^{-b} \\
& 1 & \\
& -p^{-a} & 1
\end{array}\right) \quad\left(\bmod U\left(\mathbb{Z}_{p}\right)\right) .
$$

Let $X_{a, b}^{v_{3}, v_{4}, v_{13}}(n)=\mathcal{T} * x_{a, b}^{v_{3}, v_{4}, v_{13}}$, and define

$$
S_{a, b}^{v_{3}, v_{4}, v_{13}}\left(n, \psi, \psi^{\prime}\right)=\sum_{x \in X_{a, b}^{v_{3}, v_{4}, v_{13}}(n)} \psi(u(x)) \psi^{\prime}\left(u^{\prime}(x)\right) .
$$

We also let

$$
X_{a, b}(n)=\coprod_{\substack{v_{3}, v_{4}\left(\bmod p^{r}\right) \\ v_{13}\left(\bmod p^{s}\right) \\ \text { conditions }}} X_{a, b}^{v_{3}, v_{4}, v_{13}}(n)
$$

and

$$
S_{a, b}\left(n, \psi, \psi^{\prime}\right)=\sum_{x \in X_{a, b}(n)} \psi(u(x)) \psi^{\prime}\left(u^{\prime}(x)\right)
$$


We have a partition

$$
X(n)=\coprod_{\substack{0 \leq a \leq r \\ 0 \leq b \leq s}} X_{a, b}(n)
$$

Now we consider cases $r \geq s$ and $r<s$ separately.

(i) Suppose $r>s$. As $r \geq a, r \geq s \geq b$, we see that $u(x), u^{\prime}(x)$ have entries in $p^{-r} \mathbb{Z}_{p} / \mathbb{Z}_{p}$ for all $x \in \bar{X}(n)$. Let $\mathcal{S}_{a, b}$ be a finite subset of $\mathbb{Z}_{p}^{3}$ such that

$$
X_{a, b}(n)=\coprod_{\left(v_{3}, v_{4}, v_{13}\right) \in \mathcal{S}_{a, b}} X_{a, b}^{v_{3}, v_{4}, v_{13}}(n) .
$$

By Theorem 2.4, we have

$$
S_{a, b}\left(n, \psi, \psi^{\prime}\right)=p^{-2 r}\left(1-p^{-1}\right)^{-2} \sum_{\left(v_{3}, v_{4}, v_{13}\right) \in \mathcal{S}_{a, b}}\left|X_{a, b}^{v_{3}, v_{4}, v_{13}}(n)\right| S_{w_{0}}\left(\theta_{a, b}^{v_{3}, v_{4}, v_{13}} ; r\right),
$$

where

$$
\theta_{a, b}^{v_{3}, v_{4}, v_{13}}\left(\lambda, \lambda^{\prime}\right)=\mathrm{e}\left(\frac{m_{1} \hat{v}_{2} \lambda_{1}+n_{1} p^{r-a} \lambda_{1}^{\prime}}{p^{r}}\right) \mathrm{e}\left(\frac{m_{2} \hat{v}_{14}+n_{2} p^{s-b}}{p^{s}}\right)
$$

By (4.16), we have

$$
S_{w_{0}}\left(\theta_{a, b}^{v_{3}, v_{4}, v_{13}} ; r\right)=S\left(m_{1} \hat{v}_{2}, n_{1} \hat{p}^{r-a} ; p^{r}\right) S\left(m_{2} \hat{v}_{14} p^{r-s}, n_{2} p^{r-b} ; p^{r}\right)
$$

And we obtain a bound by applying (4.1):

$$
\begin{aligned}
& \left|S_{w_{0}}\left(\theta_{a, b}^{v_{3}, v_{4}, v_{13}} ; r\right)\right| \\
& \quad \leq 4 p^{r}\left(\operatorname{gcd}\left(m_{1} \hat{v}_{2}, n_{1} p^{r-a}, p^{r}\right) \operatorname{gcd}\left(m_{2} \hat{v}_{14} p^{r-s}, n_{2} p^{r-b}, p^{r}\right)\right)^{1 / 2}
\end{aligned}
$$

(ii) Suppose $s \geq r$. Then $u(x), u^{\prime}(x)$ has entries in $p^{-s} \mathbb{Z}_{p} / \mathbb{Z}_{p}$ for all $x \in X(n)$. Again, by Theorem 2.4 we have

$$
\begin{aligned}
S_{a, b}\left(n, \psi, \psi^{\prime}\right)= & p^{-2 s}\left(1-p^{-1}\right)^{-2} \\
& \times \sum_{\left(v_{3}, v_{4}, v_{13}\right) \in \mathcal{S}_{a, b}}\left|X_{a, b}^{v_{3}, v_{4}, v_{13}}(n)\right| S_{w_{0}}\left(\theta_{a, b}^{v_{3}, v_{4}, v_{13}} ; s\right),
\end{aligned}
$$


where

$$
\begin{aligned}
& \theta_{a, b}^{v_{3}, v_{4}, v_{13}}\left(\lambda, \lambda^{\prime}\right) \\
& \quad=\mathrm{e}\left(\frac{\left(m_{1} \hat{v}_{2} p^{s-r}\right) \lambda_{1}+\left(m_{2} \hat{v}_{14}\right) \lambda_{2}+\left(n_{1} p^{s-a}\right) \lambda_{1}^{\prime}+\left(n_{2} p^{s-b}\right) \lambda_{2}^{\prime}}{p^{s}}\right) .
\end{aligned}
$$

By (4.16), we have

$$
S_{w_{0}}\left(\theta_{a, b}^{v_{3}, v_{4}, v_{13}} ; s\right)=S\left(m_{1} \hat{v}_{2} p^{s-r}, n_{1} p^{s-a} ; p^{s}\right) S\left(m_{2} \hat{v}_{14}, n_{2} p^{s-b} ; p^{s}\right) .
$$

Applying (4.1) gives

$$
\begin{aligned}
& \left|S_{w_{0}}\left(\theta_{a, b}^{v_{3}, v_{4}, v_{13}} ; s\right)\right| \\
& \quad \leq 4 p^{s}\left(\operatorname{gcd}\left(m_{1} \hat{v}_{2} p^{s-r}, n_{1} p^{s-a}, p^{s}\right), \operatorname{gcd}\left(m_{2} \hat{v}_{14}, n_{2} p^{s-b}, p^{s}\right)\right)^{1 / 2} .
\end{aligned}
$$

Now we give a bound to the size of $\mathrm{Kl}_{p}\left(n, \psi, \psi^{\prime}\right)$. To ease computation, we consider a relaxed bound by ignoring $\hat{v}_{2}$ and $\hat{v}_{14}$.

Suppose $r>s$. Then the bound says

$$
\begin{aligned}
& \left|S_{w_{0}}\left(\theta_{a, b}^{v_{3}, v_{4}, v_{13}} ; r\right)\right| \\
& \quad \leq 4 p^{r}\left(\operatorname{gcd}\left(m_{1} \hat{v}_{2}, n_{1} p^{r-a}, p^{r}\right) \operatorname{gcd}\left(m_{2} \hat{v}_{14} p^{r-s}, n_{2} p^{r-b}, p^{r}\right)\right)^{1 / 2} \\
& \quad \leq 4 p^{r}\left(\left|n_{1} n_{2}\right|_{p}^{-1} p^{2 r-a-b}\right)^{1 / 2} \\
& \quad=4 p^{2 r-\frac{a+b}{2}}\left|n_{1} n_{2}\right|_{p}^{-1 / 2} .
\end{aligned}
$$

Note that

$$
\sum_{\left(v_{3}, v_{4}, v_{13}\right) \in \mathcal{S}_{a, b}}\left|X_{a, b}^{v_{3}, v_{4}, v_{13}}(n)\right| \leq\left|\mathcal{S}_{a, b}\right| p^{a+b}
$$

Hence

$$
\begin{aligned}
\left|\mathrm{Kl}_{p}\left(n, \psi, \psi^{\prime}\right)\right| & \leq \sum_{\substack{a \leq r \\
b \leq s}}\left|S_{a, b}\left(n, \psi, \psi^{\prime}\right)\right| \\
& \leq \sum_{\substack{a \leq r \\
b \leq s}} p^{-2 r}\left(1-p^{-1}\right)^{-2} 4\left|n_{1} n_{2}\right|_{p}^{-1 / 2}\left|\mathcal{S}_{a, b}\right| p^{2 r+\frac{a+b}{2}} \\
& \ll\left|n_{1} n_{2}\right|_{p}^{-1 / 2} \sum_{\substack{a \leq r \\
b \leq s}}\left|\mathcal{S}_{a, b}\right| p^{\frac{a+b}{2}} .
\end{aligned}
$$


So it suffices to give an upper bound to $\left|\mathcal{S}_{a, b}\right|$. Such bounds were computed in [12, Sect. 5]. Note that we require $r \geq a+b$ in order to have $\mathcal{S}_{a, b}$ nonempty.

Case I: Suppose $s-r+a \geq 0$.

(a) If $s-2 r+2 a+b \geq 0$, then $\left|\mathcal{S}_{a, b}\right| \leq p^{r+s-a-b}$.

(b) If $s-2 r+2 a+b<0$, then $\left|\mathcal{S}_{a, b}\right| \leq p^{2 s-b-\left\lceil\frac{s-b}{2}\right\rceil} \leq p^{3 s / 2-b / 2}$.

Case II: Suppose $s-r+a<0$. Then $\left|\mathcal{S}_{a, b}\right| \leq p^{2 s-b-\left\lceil\frac{s-b}{2}\right\rceil} \leq p^{3 s / 2-b / 2}$.

Combining the cases, we obtain

$$
\begin{aligned}
\sum_{\substack{a \leq r \\
b \leq s}}\left|\mathcal{S}_{a, b}\right| p^{\frac{a+b}{2}} & \leq \sum_{\substack{r-s \leq a \leq r \\
2 r-2 a-s \leq b \leq r-a}} p^{r+s-\frac{a}{2}-\frac{b}{2}}+\sum_{\substack{r-s \leq a \leq r \\
b<2 r-2 a-s}} p^{\frac{3 s}{2}+\frac{a}{2}}+\sum_{\substack{a<r-s \\
b \leq s}} p^{\frac{3 s}{2}+\frac{a}{2}} \\
& \ll(s+1) p^{\frac{r}{2}+\frac{5 s}{4}} .
\end{aligned}
$$

Hence, we have for $r>s$

$$
\left|\mathrm{K}_{p}\left(n, \psi, \psi^{\prime}\right)\right| \ll\left|n_{1} n_{2}\right|_{p}^{-1 / 2}(s+1) p^{\frac{r}{2}+\frac{5 s}{4}} .
$$

For $r \leq s$, applying the same argument gives

$$
\left|\mathrm{Kl}_{p}\left(n, \psi, \psi^{\prime}\right)\right| \ll\left|n_{1} n_{2}\right|_{p}^{-1 / 2}(s-r+1) p^{r+\frac{3 s}{4}} .
$$

Combining (4.17) and (4.18), we get

$$
\left|\mathrm{Kl}_{p}\left(n, \psi, \psi^{\prime}\right)\right| \ll\left|n_{1} n_{2}\right|_{p}^{-1 / 2}(s+1) p^{\frac{r}{2}+\frac{3 s}{4}+\frac{1}{2} \min \{r, s\}} .
$$

By Proposition 3.3, we can swap the characters, so

$$
\left|\mathrm{Kl}_{p}\left(n, \psi, \psi^{\prime}\right)\right| \ll\left|m_{1} m_{2}\right|_{p}^{-1 / 2}(s+1) p^{\frac{r}{2}+\frac{3 s}{4}+\frac{1}{2} \min \{r, s\}}
$$

as well. Combining (4.19) and (4.20) yields the bound for $\mathrm{Kl}_{p}\left(n_{w_{0}, r, s}, \psi, \psi^{\prime}\right)$.

\subsection{Bounds for global Kloosterman sums}

By combining the bounds for local Kloosterman sums $\mathrm{Kl}_{p}\left(n_{w, r, s}, \psi, \psi^{\prime}\right)$, we obtain bounds for global Kloosterman sums, and prove Theorem 1.2.

Proof of Theorem 1.2 The statement for $\mathrm{Kl}\left(n_{\mathrm{id}}\left(c_{1}, c_{2}\right), \psi, \psi^{\prime}\right)$ follows because

$$
\mathrm{Kl}_{p}\left(n_{\mathrm{id}, r, s}, \psi, \psi^{\prime}\right)= \begin{cases}1 & \text { if } r=s=0 \\ 0 & \text { otherwise }\end{cases}
$$

Meanwhile, $\operatorname{Kl}\left(n_{s_{\alpha}}\left(c_{1}, 1\right), \psi, \psi^{\prime}\right)$ and $\mathrm{Kl}\left(n_{s_{\beta}}\left(1, c_{2}\right), \psi, \psi^{\prime}\right)$ are just classical Kloosterman sums. Combining local bounds for classical Kloosterman sums gives 
the global bounds, which read

$$
\begin{aligned}
& \left|\mathrm{Kl}_{p}\left(n_{s_{\alpha}}\left(c_{1}, 1\right), \psi, \psi^{\prime}\right)\right| \ll_{\varepsilon}\left(m_{1}, n_{1}, c_{1}\right)^{1 / 2} c_{1}^{1 / 2+\varepsilon} \\
& \left|\mathrm{Kl}_{p}\left(n_{s_{\beta}}\left(1, c_{2}\right), \psi, \psi^{\prime}\right)\right| \ll_{\varepsilon}\left(m_{2}, n_{2}, c_{2}\right)^{1 / 2} c_{2}^{1 / 2+\varepsilon} .
\end{aligned}
$$

For $\mathrm{Kl}\left(n_{s_{\alpha} s_{\beta}}\left(c_{1}, c_{2}\right), \psi, \psi^{\prime}\right)$ and $\mathrm{Kl}\left(n_{s_{\beta} s_{\alpha}}\left(c_{1}, c_{2}\right), \psi, \psi^{\prime}\right)$, we again combine the local bounds given in Theorem 1.1 yields the global bounds.

For $\operatorname{Kl}\left(n_{s_{\alpha} s_{\beta} s_{\alpha}}\left(c_{1}, c_{2}\right), \psi, \psi^{\prime}\right)$ and $\operatorname{Kl}\left(n_{s_{\beta} s_{\alpha} s_{\beta}}\left(c_{1}, c_{2}\right), \psi, \psi^{\prime}\right)$, the situation is more complicated, since the shapes of the local bounds depend on the relative size of $r, s$. Therefore, in order to obtain a global bound, we have to find an expression for the local bound that works for all values of $r, s$.

We start with $\operatorname{Kl}\left(n_{s_{\alpha} s_{\beta} s_{\alpha}}\left(c_{1}, c_{2}\right), \psi, \psi^{\prime}\right)$. For $s \leq r$, we have

$$
\begin{aligned}
\left|\mathrm{Kl}_{p}\left(n_{s_{\alpha} s_{\beta} s_{\alpha}, r, s}, \psi, \psi^{\prime}\right)\right| & \ll p^{\frac{r}{3}+\frac{4 s}{3}+\frac{2}{3} \min \left\{\operatorname{ord}_{p}\left(m_{1}\right), \operatorname{ord}_{p}\left(n_{1}\right)\right\}+\frac{1}{3} \operatorname{ord}_{p}\left(m_{2}\right)} \\
& \leq p^{\frac{4 r}{3}+\frac{s}{3}+\frac{2}{3} \min \left\{\operatorname{ord}_{p}\left(m_{1}\right), \operatorname{ord}_{p}\left(n_{1}\right)\right\}+\frac{1}{3} \operatorname{ord}_{p}\left(m_{2}\right)} .
\end{aligned}
$$

For $r<s<2 r$, we have

$$
\left|\mathrm{Kl}_{p}\left(n_{s_{\alpha} s_{\beta} s_{\alpha}, r, s}, \psi, \psi^{\prime}\right)\right| \ll p^{r+\operatorname{ord}_{p}\left(m_{2}\right)}+p^{r+\frac{s}{2}+\min \left\{\operatorname{ord}_{p}\left(m_{1}\right), \operatorname{ord}_{p}\left(n_{1}\right)\right\}},
$$

and we have inequalities

$$
\begin{aligned}
& p^{r+\operatorname{ord}_{p}\left(m_{2}\right)}+p^{r+\frac{s}{2}+\min \left\{\operatorname{ord}_{p}\left(m_{1}\right), \operatorname{ord}_{p}\left(n_{1}\right)\right\}} \\
& \quad \leq p^{r+\operatorname{ord}_{p}\left(m_{2}\right)}+p^{\frac{4 r}{3}+\frac{s}{3}+\min \left\{\operatorname{ord}_{p}\left(m_{1}\right), \operatorname{ord}_{p}\left(n_{1}\right)\right\}}, \\
& p^{r+\operatorname{ord}_{p}\left(m_{2}\right)}+p^{r+\frac{s}{2}+\min \left\{\operatorname{ord}_{p}\left(m_{1}\right), \operatorname{ord}_{p}\left(n_{1}\right)\right\}} \\
& \quad \leq p^{s+\operatorname{ord}_{p}\left(m_{2}\right)}+p^{\frac{r}{6}+\frac{4 s}{3}+\min \left\{\operatorname{ord}_{p}\left(m_{1}\right), \operatorname{ord}_{p}\left(n_{1}\right)\right\}} .
\end{aligned}
$$

For $s=2 r$, we have

$$
\left|\mathrm{K}_{p}\left(n_{s_{\alpha} s_{\beta} s_{\alpha}, r, s}, \psi, \psi^{\prime}\right)\right| \ll p^{r+\operatorname{ord}_{p}\left(m_{2}\right)}=p^{\frac{s}{2}+\operatorname{ord}_{p}\left(m_{2}\right)} .
$$

So we can conclude for $0 \leq s \leq 2 r$ that

$$
\left|\mathrm{Kl}_{p}\left(n_{s_{\alpha} s_{\beta} s_{\alpha}, r, s}, \psi, \psi^{\prime}\right)\right| \ll p^{\min \left\{\frac{4 r}{3}+\frac{s}{3}, \frac{r}{3}+\frac{4 s}{3}\right\}+\operatorname{ord}_{p}\left(m_{2}\right)+\min \left\{\operatorname{ord}_{p}\left(m_{1}\right), \operatorname{ord}_{p}\left(n_{1}\right)\right\}} .
$$

Since we may assume from (4.6) that ord $\left(m_{1}\right), \operatorname{ord}_{p}\left(n_{1}\right) \leq r$, and $\operatorname{ord}_{p}\left(m_{2}\right) \leq s$, we have

$$
\left|\mathrm{Kl}\left(n_{s_{\alpha} s_{\beta} s_{\alpha}}\left(c_{1}, c_{2}\right), \psi, \psi^{\prime}\right)\right| \ll_{\varepsilon}\left(m_{1}, n_{1}, c_{1}\right)\left(m_{2}, c_{2}\right)\left(c_{1}, c_{2}\right)\left(c_{1} c_{2}\right)^{1 / 3+\varepsilon}
$$

for every $\varepsilon>0$. 
Now we consider $\operatorname{Kl}\left(n_{s_{\beta} s_{\alpha} s_{\beta}}\left(c_{1}, c_{2}\right), \psi, \psi^{\prime}\right)$. For $r \leq s / 2$, we have

$$
\begin{aligned}
\left|\mathrm{Kl}_{p}\left(n_{s_{\beta} s_{\alpha} s_{\beta}, r, s}, \psi, \psi^{\prime}\right)\right| & \ll p^{\frac{3 r}{2}+\frac{s}{2}+\frac{1}{2} \operatorname{ord}_{p}\left(m_{1}\right)+\frac{1}{2} \min \left\{\operatorname{ord}_{p}\left(m_{2}\right), \operatorname{ord}_{p}\left(n_{2}\right)\right\}} \\
& \leq p^{-\frac{r}{2}+\frac{3 s}{2}+\frac{1}{2} \operatorname{ord}_{p}\left(m_{1}\right)+\frac{1}{2} \min \left\{\operatorname{ord}_{p}\left(m_{2}\right), \operatorname{ord}_{p}\left(n_{2}\right)\right\}} .
\end{aligned}
$$

For $s / 2<r<s$, we have

$$
\begin{aligned}
\left|\mathrm{Kl}_{p}\left(n_{s_{\beta} s_{\alpha} s_{\beta}, r, s}, \psi, \psi^{\prime}\right)\right| & \ll p^{-\frac{r}{2}+\frac{3 s}{2}+\frac{1}{2} \operatorname{ord}_{p}\left(m_{1}\right)+\frac{1}{2} \min \left\{\operatorname{ord}_{p}\left(m_{2}\right), \operatorname{ord}_{p}\left(n_{2}\right)\right\}} \\
& \leq p^{\frac{3 r}{2}+\frac{s}{2}+\frac{1}{2} \operatorname{ord}_{p}\left(m_{1}\right)+\frac{1}{2} \min \left\{\operatorname{ord}_{p}\left(m_{2}\right), \operatorname{ord}_{p}\left(n_{2}\right)\right\}}
\end{aligned}
$$

For $s=r$, we have

$$
\left|\mathrm{Kl}_{p}\left(n_{s_{\beta} s_{\alpha} s_{\beta}, r, s}, \psi, \psi^{\prime}\right)\right| \ll p^{s+\min \left\{\operatorname{ord}_{p}\left(m_{1}\right), \operatorname{ord}_{p}\left(n_{2}\right)\right\}}=p^{r+\min \left\{\operatorname{ord}_{p}\left(m_{1}\right), \operatorname{ord}_{p}\left(n_{2}\right)\right\}} .
$$

So we can conclude for $0 \leq r \leq s$ that

$$
\left|\mathrm{Kl}_{p}\left(n_{s_{\beta} s_{\alpha} s_{\beta}, r, s}, \psi, \psi^{\prime}\right)\right| \ll p^{\min \left\{\frac{3 r}{2}+\frac{s}{2},-\frac{r}{2}+\frac{3 s}{2}\right\}+\operatorname{ord}_{p}\left(m_{1}\right)+\frac{1}{2} \min \left\{\operatorname{ord}_{p}\left(m_{2}\right), \operatorname{ord}_{p}\left(n_{2}\right)\right\}} .
$$

Since we may assume from (4.11) that ord $\left.\operatorname{ord}_{1}\right) \leq r$, and $\operatorname{ord}_{p}\left(m_{2}\right), \operatorname{ord}_{p}\left(n_{2}\right) \leq s$, we have

$$
\left|\mathrm{Kl}\left(n_{s_{\beta} s_{\alpha} s_{\beta}}\left(c_{1}, c_{2}\right), \psi, \psi^{\prime}\right)\right| \ll_{\varepsilon}\left(m_{1}, c_{1}\right)\left(m_{2}, n_{2}, c_{2}\right)\left(c_{1}^{2}, c_{2}\right) c_{1}^{-1 / 2} c_{2}^{1 / 2}\left(c_{1} c_{2}\right)^{\varepsilon}
$$

for every $\varepsilon>0$.

For $\operatorname{Kl}\left(n_{w_{0}}\left(c_{1}, c_{2}\right), \psi, \psi^{\prime}\right)$, the local bound again consists of a single expression, so the local bounds given in Theorem 1.1 can be combined directly to give the stated global bound.

\section{Symplectic Poincaré series}

In this section, we compute the Fourier coefficients of symplectic Poincaré series, in terms of auxiliary Kloosterman sums.

Definition (a) Let $n \in N\left(\mathbb{Q}_{p}\right)$, and $\psi_{p}, \psi_{p}^{\prime}$ be characters of $U\left(\mathbb{Q}_{p}\right)$ which are trivial on $U\left(\mathbb{Z}_{p}\right)$. Then the local auxiliary Kloosterman sum is defined to be

$$
\underline{\mathrm{Kl}}_{p}\left(n, \psi_{p}, \psi_{p}^{\prime}\right)=\sum_{\substack{x \in X(n) \\ x=b_{1} n b_{2}}} \psi_{p}\left(b_{1}\right) \psi_{p}^{\prime}\left(b_{2}\right)
$$

if $\psi_{p}\left(n u n^{-1}\right)=\psi_{p}^{\prime}(u)$ for $u \in \bar{U}_{n}\left(\mathbb{Q}_{p}\right)$, and zero otherwise. We say $\underline{\mathrm{Kl}}_{p}\left(n, \psi_{p}, \psi_{p}^{\prime}\right)$ is well-defined if $\psi_{p}\left(n u n^{-1}\right)=\psi_{p}^{\prime}(u)$ for $u \in \bar{U}_{n}\left(\mathbb{Q}_{p}\right)$. 
(b) Let $n \in N(\mathbb{Q})$, and $\psi=\prod_{p} \psi_{p}, \psi^{\prime}=\prod_{p} \psi_{p}^{\prime}$ be characters of $U(\mathbb{A})$ which are trivial on $\prod_{p} U\left(\mathbb{Z}_{p}\right)$. Then the global auxiliary Kloosterman sum is defined to be

$$
\underline{\mathrm{Kl}}\left(n, \psi, \psi^{\prime}\right)=\prod_{p} \underline{\mathrm{Kl}}_{p}\left(n, \psi_{p}, \psi_{p}^{\prime}\right) .
$$

We first show that the auxiliary Kloosterman sums are well-defined.

Proposition 5.1 [9, Proposition 1.3] Let $G=\operatorname{Sp}\left(2 r, \mathbb{Q}_{p}\right), n \in N\left(\mathbb{Q}_{p}\right)$, and $x \in$ $X(n)$, with Bruhat decomposition $x=b_{1} n b_{2}$, with $b_{1}, b_{2} \in U\left(\mathbb{Q}_{p}\right)$. Let $\psi, \psi^{\prime}$ be characters of $U\left(\mathbb{Q}_{p}\right)$ which are trivial on $U\left(\mathbb{Z}_{p}\right)$. Then the quantity $\psi\left(b_{1}\right) \psi^{\prime}\left(b_{2}\right)$ is well-defined as a function on $X(n)$ if $\psi\left(n u n^{-1}\right)=\psi^{\prime}(u)$ for $u \in \bar{U}_{n}\left(\mathbb{Q}_{p}\right)$.

Proof Suppose $\psi\left(n u n^{-1}\right)=\psi^{\prime}(u)$ for all $u \in \bar{U}_{n}\left(\mathbb{Q}_{p}\right)$. Let $x=b_{1} n b_{2}=b_{1}^{\prime} n b_{2}^{\prime}$ be two Bruhat decompositions. This says $b_{1}^{\prime}=\gamma b_{1}$ for some $\gamma \in U\left(\mathbb{Z}_{p}\right)$, and $b_{2}^{\prime}=b_{2} \delta$ for some $\delta \in U_{n}\left(\mathbb{Z}_{p}\right)$. Then we have

$$
U\left(\mathbb{Z}_{p}\right) b_{1} n b_{2} \delta^{-1}=U\left(\mathbb{Z}_{p}\right) b_{1} n b_{2},
$$

which implies $b_{2} b_{2}^{\prime-1}=b_{2} \delta^{-1} b_{2}^{-1} \in \bar{U}_{n}\left(\mathbb{Q}_{p}\right)$. Now, from the equivalence of Bruhat decompositions, we deduce that

$$
U\left(\mathbb{Z}_{p}\right) n b_{2} b_{2}^{\prime-1} n^{-1} U_{n}\left(\mathbb{Z}_{p}\right)=U\left(\mathbb{Z}_{p}\right) b_{1}^{-1} b_{1}^{\prime} U_{n}\left(\mathbb{Z}_{p}\right)
$$

which implies $\psi^{\prime}\left(b_{2} b_{2}^{\prime-1}\right)=\psi\left(n b_{2} b_{2}^{\prime-1} n^{-1}\right)=\psi\left(b_{1}^{-1} b_{1}^{\prime}\right)$.

Proposition 5.2 If $\underline{\mathrm{Kl}}_{p}\left(n, \psi_{p}, \psi_{p}^{\prime}\right)$ is well-defined, then $\underline{\mathrm{Kl}}_{p}\left(n, \psi_{p}, \psi_{p}^{\prime}\right)=$ $\mathrm{Kl}_{p}\left(n, \psi_{p}, \psi_{p}^{\prime}\right)$.

\section{Proof Trivial.}

The Fourier coefficients $P_{\psi, \psi^{\prime}}(g)$ can be evaluated using the following theorem of Friedberg:

Theorem 5.3 [9, Theorem A] The Fourier coefficient $P_{\psi, \psi^{\prime}}(g)$ of $\operatorname{Sp}(2 r)$ Poincaré series is given by

$$
P_{\psi, \psi^{\prime}}(g)=\sum_{w \in W} \sum_{\substack{n \in N(\mathbb{Q}) \\ w(n)=w}} \underline{\mathrm{Kl}}\left(n, \psi, \psi^{\prime}\right) \int_{U_{w}(\mathbb{R})} \mathcal{F}_{\psi}\left(n u_{1} y\right) \overline{\psi^{\prime}}\left(u_{1}\right) d u_{1} .
$$

Remark In [9], the statement concerns GL( $r)$ Poincaré series, but the proof also works for $\operatorname{Sp}(2 r)$ Poincaré series. 


\subsection{Sp(4) Poincaré series}

Let $G=\operatorname{Sp}\left(4, \mathbb{Q}_{p}\right)$, and $\psi=\psi_{m_{1}, m_{2}}, \psi^{\prime}=\psi_{n_{1}, n_{2}}$. We give a table of conditions for auxiliary $\mathrm{Sp}(4)$ Kloosterman sums $\underline{\mathrm{Kl}}_{p}\left(n_{w, r, s}, \psi, \psi^{\prime}\right)$ to be well-defined.

\begin{tabular}{llll}
\hline $\mathrm{w}$ & Well-definedness conditions & $\mathrm{w}$ & Well-definedness conditions \\
\hline $\mathrm{id}$ & $m_{1}=n_{1}, m_{2}=n_{2}$ & $s_{\beta} s_{\alpha}$ & $m_{1}=n_{2}=0$ \\
$s_{\alpha}$ & $m_{2}=n_{2}=0$ & $s_{\alpha} s_{\beta} s_{\alpha}$ & $n_{2}=m_{2} p^{2 r-2 s}$ \\
$s_{\beta}$ & $m_{1}=n_{1}=0$ & $s_{\beta} s_{\alpha} s_{\beta}$ & $n_{1}=m_{1} p^{s-2 r}$ \\
$s_{\alpha} s_{\beta}$ & $m_{2}=n_{1}=0$ & $w_{0}$ & - \\
\hline
\end{tabular}

Remark From this table, we see that not all Kloosterman sums $\mathrm{Kl}_{p}\left(n, \psi, \psi^{\prime}\right)$ correspond to a well-defined auxiliary Kloosterman $\operatorname{sum~} \underline{\mathrm{Kl}}_{p}\left(n, \psi, \psi^{\prime}\right)$.

From the well-definedness conditions for $\underline{\mathrm{Kl}}_{p}\left(n_{w, r, s}, \psi, \psi^{\prime}\right)$, we see that when $\psi=\psi_{m_{1}, m_{2}}, \psi^{\prime}=\psi_{n_{1}, n_{2}}$ are non-degenerate, i.e. $m_{1} m_{2}, n_{1} n_{2} \neq 0$, then

$$
w=\mathrm{id}, s_{\alpha} s_{\beta} s_{\alpha}, s_{\beta} s_{\alpha} s_{\beta}, w_{0} \in W
$$

are the only Weyl elements that contribute to the Fourier coefficient $P_{\psi, \psi^{\prime}}(g)$.

Acknowledgements The author would like to thank Valentin Blomer for his guidance on the project.

Funding Open Access funding enabled and organized by Projekt DEAL.

Open Access This article is licensed under a Creative Commons Attribution 4.0 International License, which permits use, sharing, adaptation, distribution and reproduction in any medium or format, as long as you give appropriate credit to the original author(s) and the source, provide a link to the Creative Commons licence, and indicate if changes were made. The images or other third party material in this article are included in the article's Creative Commons licence, unless indicated otherwise in a credit line to the material. If material is not included in the article's Creative Commons licence and your intended use is not permitted by statutory regulation or exceeds the permitted use, you will need to obtain permission directly from the copyright holder. To view a copy of this licence, visit http://creativecommons.org/licenses/by/4.0/.

\section{References}

1. Adolphson, A., Sperber, S.: Expoonential sums and Newton polyhedra: cohomology and estimates. Ann. Math. 130(2), 367-406 (1989)

2. Bump, D., Friedberg, S., Goldfeld, D.: Poincaré series and Kloosterman sums for SL(3, $\mathbb{Z})$. Acta Arith. 50, 31-89 (1988)

3. Bump, D., Friedberg, S., Hoffstein, J.: Eisenstein series on the metaplectic group and nonvanishing theorems for automorphic L-functions and their derivatives. Ann. Math. 131, 53-127 (1990)

4. Blomer, V.: The relative trace formula in analytic number theory. To appear in Simons Symp. https:// arxiv.org/abs/1912.08137 (2019)

5. Dąbrowski, R.: Kloosterman sums for Chevalley groups. Trans. Am. Math. Soc. 337, 757-769 (1993)

6. Deligne, P.: Cohomologie étale (SGA 4 1/2). Lecture Notes in Mathematics, vol. 569. Springer, Berlin (1977) 
7. Dạbrowski, R., Fisher, B.: A stationary phase formula for exponential sums over $\mathbb{Z} / p^{m} \mathbb{Z}$ and applications to GL(3)-Kloosterman sums. Acta Arith. 80, 1-48 (1997)

8. Dąbrowski, R., Reeder, M.: Kloosterman sets in reductive groups. J. Number Theory 73, 228-255 (1998)

9. Friedberg, S.: Poincaré series for GL(n): Fourier expansion, Kloosterman sums, and algebreogeometric estimates. Math. Z. 196, 165-188 (1987)

10. Goldfeld, D., Stade, E., Woodbury, M.: An orthogonality relation for GL $(4, R)$. In preprint. https:// arxiv.org/abs/1910.13586 (2019)

11. Kitaoka, Y.: Fourier coefficients of Siegel cusp forms of degree two. Nagoya Math. J. 93, 149-171 (1984)

12. Man, S.H.: Symplectic Eisenstein series. In preprint. https://arxiv.org/abs/2003.06890 (2020)

13. Smith, R.A.: Estimates of exponential sums. Proc. Am. Math. Soc. 79(3), 365-368 (1980)

14. Stevens, G.: Poincaré series on GL $(r)$ and Kloostermann sums. Math. Ann. 277, 25-51 (1987)

15. Tóth, Á.: Symplectic Kloosterman sums. Studia Sci. Math. Hungar. 50, 143-158 (2013)

16. Weil, A.: On some exponential sums. Proc. Natl. Acad. Sci. 34, 204-207 (1948)

Publisher's Note Springer Nature remains neutral with regard to jurisdictional claims in published maps and institutional affiliations. 